\title{
Generalized Variational Principles, Global Weak Solutions and Behavior with Random Initial Data for Systems of Conservation Laws Arising in Adhesion Particle Dynamics
}

\author{
Weinan E ${ }^{1}$, Yu.G. Rykov ${ }^{2}$, Ya.G. Sinai ${ }^{3}$ \\ ${ }^{1}$ Courant Institute of Mathematical Sciences, New York, NY 10012, USA \\ ${ }^{2}$ Keldysh Institute of Applied Mathematics of Russian Academy of Sciences, Moscow, Russia \\ ${ }^{3}$ Mathematics Department, Princeton University, Princeton, NJ 08544, USA and Landau Institute \\ of Theoretical Physics, Moscow, Russia
}

Received: 30 December 1994/in revised form: 1 May 1995

\begin{abstract}
We study systems of conservation laws arising in two models of adhesion particle dynamics. The first is the system of free particles which stick under collision. The second is a system of gravitationally interacting particles which also stick under collision. In both cases, mass and momentum are conserved at the collisions, so the dynamics is described by $2 \times 2$ systems of conservations laws. We show that for these systems, global weak solutions can be constructed explicitly using the initial data by a procedure analogous to the Lax-Oleinik variational principle for scalar conservation laws. However, this weak solution is not unique among weak solutions satisfying the standard entropy condition. We also study a modified gravitational model in which, instead of momentum, some other weighted velocity is conserved at collisions. For this model, we prove both existence and uniqueness of global weak solutions. We then study the qualitative behavior of the solutions with random initial data. We show that for continuous but nowhere differentiable random initial velocities, all masses immediately concentrate on points even though they were continuously distributed initially, and the set of shock locations is dense.
\end{abstract}

\section{Introduction}

This paper has two main goals: The first is to give an explicit construction of weak solutions for the initial value problem of the systems of conservation laws:

$$
\left\{\begin{aligned}
\rho_{t}+(\rho u)_{x} & =0 \\
(\rho u)_{t}+\left(\rho u^{2}\right)_{x} & =0
\end{aligned}\right.
$$

and

$$
\left\{\begin{aligned}
\rho_{t}+(\rho u)_{x} & =0 \\
(\rho u)_{t}+\left(\rho u^{2}\right)_{x} & =-\rho g_{x} \\
g_{x x} & =\rho .
\end{aligned}\right.
$$

The second is to study the qualitative behavior of such weak solutions when initial data are random. We prove that for a wide class of probability distributions for the 
initial data, almost every weak solution has the following structure: At any positive time $t>0, \rho(\cdot, t)$ becomes a purely singular measure even though it may be continuous at $t=0$. Moreover, this singular measure is supported on a dense set which can also be considered as the shock set of $u$. We will also study a variant of (1.2) in which a weighted velocity instead of momentum is conserved at the collisions [GMS, VFDN]:

$$
\left\{\begin{aligned}
\rho_{t}+(\rho u)_{x} & =0 \\
u_{t}+\left(\frac{u^{2}}{2}\right)_{x} & =-g_{x} \\
g_{x x} & =\rho .
\end{aligned}\right.
$$

Our construction of weak solutions for (1.1) is based on a connection between (1.1) and the "sticky particle model" of Zeldovich (see [Z] and also [CPY]). There is a similar connection between (1.2) and the gravitationally interacting sticky particles. Consider a system of particles on $R^{1}$ with initial velocities $\left\{v_{j}^{0}\right\}$, locations $\left\{x_{j}^{0}\right\}$ and masses $\left\{m_{j}^{0}\right\}, j \in \mathbf{Z}$. The particles move with constant velocities unless they collide. At collisions the colliding particles stick and form a new massive particle. The mass and velocity of this new particle are given by the laws of conservation of mass and momentum. This model was proposed by Zeldovich [SZ], and developed further by Kofman, Shandarin, et al. (see [GMS, KPS], and the survey paper [VDFN]) to explain the formation of large scale structures in the universe. In this context it is also referred to as the model of "adhesion dynamics." One main result of this paper is that the adhesion dynamics of free particles is in a sense integrable, and this gives rise to weak solutions of (1.1).

A similar connection exists between (1.2) and the gravitationally interacting sticky particles. The Hamiltonian governing the dynamics between collisions is given by

$$
H(p, x)=\sum_{i} \frac{p_{i}^{2}}{2 m_{i}}+\sum_{i \neq j} m_{i} m_{j}\left|x_{i}-x_{j}\right| .
$$

We will assume that $\sum_{i} m_{i}<\infty$. When particles collide, again they form a new particle with mass and velocity given by the conservation of mass and momentum. The gravitational force acting on a particle is proportional to the difference between the total masses from the right and from the left of the particle. This system is also integrable in the same sense and leads to weak solutions of (1.2).

For smooth solutions, (1.1) is equivalent to the Burgers equation

$$
u_{t}+\left(\frac{u^{2}}{2}\right)_{x}=0
$$

together with a scalar transport equation

$$
\rho_{t}+(\rho u)_{x}=0 \text {. }
$$

Given the initial data $\left\{\rho_{0}, u_{0}\right\}$, the solution of (1.5)-(1.6) can be easily found via the method of characteristics. Define the forward flow map $\varphi_{t}: R^{1} \rightarrow R^{1}$ by

$$
x=\varphi_{t}(y)=y+t u_{0}(y) .
$$

For small $t$, this map is usually invertible, and we have

$$
u(x, t)=u_{0}\left(\varphi_{t}^{-1}(x)\right), \quad \rho(x, t)=\rho_{0}\left(\varphi_{t}^{-1}(x)\right)\left|\frac{\partial x}{\partial y}\right|^{-1},
$$

where $y=\varphi_{t}^{-1}(x)$ defines the backward flow map. 
It is well-known [L] that this construction ceases to be valid after some critical time $T^{*}$ at which the solution of (1.5) develops shocks. In general (1.1) and (1.5)-(1.6) also cease to be equivalent after $T^{*}$.

In analogy with fluid mechanics, we call $y$ the Lagrangian coordinate and $\varphi_{t}(y)$ the Eulerian coordinate at time $t$. After $T^{*}$ the mapping $y \rightarrow \varphi_{t}(y)$ is no longer one-to-one, and no longer defined by (1.7): a whole interval can be mapped to a single point which is the location of a shock.

However, in all cases $\varphi_{t}$ defines a partition $\xi_{t}$ of $R^{1}$ where elements of the partition are given by

$$
\mathscr{D}_{t}(x)=\left\{\varphi_{t}^{-1}(x), x \in R^{1}\right\} .
$$

We should stress that the solutions are assumed to be continuous from the right. The elements of $\xi_{t}$ can be either single points, or segments. More importantly, knowing $\xi_{t}$, we can reconstruct $\varphi_{t}$ and $u(\cdot, t)$ from the two conservation laws:

$$
\varphi_{t}(y)=\frac{\int_{C_{t}(y)}\left(\eta+t u_{0}(\eta)\right) \rho_{0}(\eta) d \eta}{\int_{C_{t}(y)} \rho_{0}(\eta) d \eta}, \quad u(x, t)=\frac{\int_{\mathscr{D}_{t}(x)} u_{0}(\eta) \rho_{0}(\eta) d \eta}{\int_{\mathscr{D}_{t}(x)} \rho_{0}(\eta) d \eta},
$$

where $C_{t}(y)$ denotes the element of the partition $\xi_{t}$ containing $y$, and $\mathscr{D}_{t}(x)=$ $\varphi_{t}^{-1}(x)$. In the more general case when the initial distribution of mass is given by a nonnegative Borel measure $P_{0},(1.10)$ takes the form

$$
\varphi_{t}(y)=\frac{\int_{C_{t}(y)}\left(\eta+t u_{0}(\eta)\right) d P_{0}(\eta)}{\int_{C_{t}(y)} d P_{0}(\eta)}, \quad u(x, t)=\frac{\int_{\mathscr{D}_{t}(x)} u_{0}(\eta) d P_{0}(\eta)}{\int_{\mathscr{D}_{t}(x)} d P_{0}(\eta)} .
$$

Both (1.10) and (1.11) state that $\varphi_{t}(y)$ is now the position of the center of mass of $C_{t}(y)$.

We are left with the key step of defining $\left\{\xi_{t}\right\}_{t>0}$. Let us first consider the simpler case of a finite number of particles with initial data $\left\{x_{j}^{0}, v_{j}^{0}, m_{j}^{0}\right\}, 1 \leqq j \leqq N$. A crucial observation is that the necessary and sufficient condition for $N$ particles to collide and form a single particle before, or at time $t$, is that

$$
\frac{\sum_{j=1}^{J}\left(x_{j}^{0}+t v_{j}^{0}\right) m_{j}^{0}}{\sum_{j=1}^{J} m_{j}^{0}} \geqq \frac{\sum_{j=J+1}^{N}\left(x_{j}^{0}+t v_{j}^{0}\right) m_{j}^{0}}{\sum_{j=J+1}^{N} m_{j}^{0}}
$$

holds for all $J, 1 \leqq J \leqq N-1$. Indeed assume that (1.12) holds, yet there are more than one cluster of particles at time $t$. Without loss of generality, let us assume that there are two such clusters, $\left\{1,2, \ldots, J^{\prime}\right\}$ and $\left\{J^{\prime}+1, \ldots, N\right\}$ located at $X_{1}(t)$ and $X_{2}(t)$ with $X_{1}(t)<X_{2}(t)$. Then we have

$$
X_{1}(t)=\frac{\sum_{j=1}^{J^{\prime}}\left(x_{j}^{0}+t v_{j}^{0}\right) m_{j}^{0}}{\sum_{j=1}^{J^{\prime}} m_{j}^{0}}<X_{2}(t)=\frac{\sum_{j=J^{\prime}+1}^{N}\left(x_{j}^{0}+t v_{j}^{0}\right) m_{j}^{0}}{\sum_{j=J^{\prime}+1}^{N} m_{j}^{0}},
$$

since the conservation of mass and momentum dictates that the cluster has to be located at the center of mass. Equation (1.13) contradicts the assumption that (1.12) holds for all $j$. On the other hand, assume that (1.12) is violated for some $J=J^{\prime}$, then the group of particles $\left\{1, \ldots, J^{\prime}\right\}$ will never catch up with the group $\left\{J^{\prime}+\right.$ $1, \ldots, N\}$ before time $t$. For details, see Sect. 3 .

Before going into the continuous case, let us state the conditions we will impose on the initial data. 
Let $P_{0}, I_{0} \in M$ : the space of Radon measures on $R^{1}, P_{0} \geqq 0$.

(A1) $P_{0}(\Delta)<\infty$ for any compact $\Delta \subset R^{1}$ and $P_{0}$ is either discrete or absolutely continuous with respect to the Lebesgue measure. In the latter case, we assume that density $\rho_{0}(x)>0$, for $x \in \operatorname{Supp}\left(P_{0}\right)$. If $\operatorname{Supp}\left(P_{0}\right)$ is unbounded, we assume additionally

$$
\int_{0}^{x} s d P_{0}(s) \rightarrow+\infty \quad \text { as }|x| \rightarrow+\infty \text {. }
$$

(A2) The initial distribution of momentum $I_{0}$ is absolutely continuous with respect to $P_{0}$. The Radon-Nikodym derivative $u(\cdot, 0)=\frac{d I_{0}}{d P_{0}}$ is the initial velocity. In the case when $P_{0}$ is absolutely continuous, we assume that $u(\cdot, 0)$ is also continuous.

(A3) For any $z>0$

$$
\sup _{|x| \leqq z}\left|u_{0}(x)\right| \leqq b_{0}(z) \text { and } \lim _{|z| \rightarrow \infty} \frac{1}{z} b_{0}(z)=0 .
$$

The first essential result of this paper is the following principle for the construction of $\xi_{t}$ using the initial data.

Generalized Variational Principle (GVP): $y \in R^{1}$ is the left endpoint of an element of $\xi_{t}$ iff for any $y^{-}, y^{+} \in R^{1}$, such that $y^{-}<y<y^{+}$, the following holds:

$$
\frac{\int_{\left[y^{-}, y\right)}(\eta+t u(\eta ; 0)) d P_{0}(\eta)}{\int_{\left[y^{-}, y\right)} d P_{0}(\eta)}<\frac{\int_{\left[y, y^{+}\right]}(\eta+t u(\eta ; 0)) d P_{0}(\eta)}{\int_{\left[y, y^{+}\right]} d P_{0}(\eta)} .
$$

We can also formulate GVP for right endpoints of elements of $\xi_{t}$, but we will omit this since we do not need it.

Having $\left\{\xi_{t}\right\}_{t>0}$, we define $\varphi_{t}$ via $(1.11)$ and the density and momentum distributions at time $t, P_{t}$ and $I_{t}$, by

$$
P_{t}(\Delta)=P_{0}\left(\varphi_{t}^{-1}(\Delta)\right), \quad I_{t}(\Delta)=I_{0}\left(\varphi_{t}^{-1}(\Delta)\right)
$$

for $\Delta \subset R^{1}$. In the case of continuous $u(x ; 0)$ the mapping $\varphi_{t}$ is also continuous. It is clear that $I_{t}$ is absolutely continuous with respect to $P_{t}$, and we can introduce the Radon-Nikodym derivative

$$
u(x, t)=\frac{d I_{t}}{d P_{t}}(x)
$$

which is the velocity at $(x, t)$.

We will use the following definition of weak solutions.

Definition 1. Let $\left(P_{t}, I_{t}\right)$ be a family of Borel measures, weakly continuous with respect to $t$, such that $I_{t}$ is absolutely continuous with respect to $P_{t}$ for each fixed $t$. Define $u$ via (1.16). $\left(P_{t}, I_{t}, u\right)_{t \geqq 0}$ is a weak solution of (1.1) if, for any $f, g \in C_{0}^{1}\left(R^{1}\right)$, the space of $C^{1}$-functions on $R^{1}$ with compact support, and $0<t_{1}<t_{2}$,

$$
\begin{gathered}
\int f(\eta) d P_{t_{2}}(\eta)-\int f(\eta) d P_{t_{1}}(\eta)=\int_{t_{1}}^{t_{2}} d \tau \int f^{\prime}(\eta) d I_{\tau}(\eta), \\
\int g(\eta) d I_{t_{2}}(\eta)-\int g(\eta) d I_{t_{1}}(\eta)=\int_{t_{1}}^{t_{2}} d \tau \int g^{\prime}(\eta) u(\eta, \tau) d I_{\tau}(\eta) .
\end{gathered}
$$


Theorem 1. Under the assumptions (A1-3), the family $\left(P_{t}, I_{t}, u\right)_{t \geqq 0}$ constructed using GVP gives a weak solution of $(1.1)$ with initial data $\left(P_{0}, I_{0}\right)$ in the sense that

$$
\lim P_{t}=P_{0}, \quad \lim I_{t}=I_{0}
$$

weakly as $t \rightarrow 0^{+}$.

Next we turn to (1.2). First of all, we remark that the third equation in (1.2) can be interpreted as

$$
-g_{x}=\left(\int_{x}^{+\infty} \rho(\xi, t) d \xi-\int_{-\infty}^{x} \rho(\xi, t) d \xi\right),
$$

i.e. the acceleration at a point is proportional to the difference between the total masses from the right and from the left of that point. For the initial data, in addition to (A1-A3) we will also assume

(A4) $P_{0}\left(R^{1}\right)<+\infty$.

The characteristics of (1.2) are now given by quadratic functions of $t$ :

$$
x(t)=y+u_{0}(y) t+\frac{1}{2} a_{0}(y) t^{2} .
$$

This has the effect of changing (1.11) to

$$
\begin{aligned}
& \varphi_{t}(y)=\frac{\int_{C_{t}(y)}\left(\eta+t u_{0}(\eta)\right) d P_{0}(\eta)}{\int_{C_{t}(y)} d P_{0}(\eta)}+a\left(C_{t}(y)\right) \frac{t^{2}}{2}, \\
& u(x, t)=\frac{\int_{\mathscr{D}_{t}(x)} u_{0}(\eta) d P_{0}(\eta)}{\int_{\mathscr{D}_{t}(x)} d P_{0}(\eta)}+a\left(\mathscr{D}_{t}(x)\right) t,
\end{aligned}
$$

where

$$
a\left(C_{t}(y)\right)=P_{0}\left(I^{+}\right)-P_{0}\left(I^{-}\right) .
$$

$I^{+}$and $I^{-}$are respectively the right and left connected component of $R^{1} \backslash C_{t}(y)$.

As before, the key issue is to construct the family of partitions of $R^{1},\left\{\xi_{t}\right\}_{t \geq 0}$. In this case we formulate an analogous Generalized Variational Principle (GVP): $y \in R^{1}$ is the left end point of an element of $\xi_{t}$ iff for any $y^{+}, y^{-}, y^{-}<y<y^{+}$, the following holds:

$$
\begin{aligned}
& \frac{\int_{\left[y^{-}, y\right)}\left(\eta+t u_{0}(\eta)\right) d P_{0}(\eta)}{\int_{\left[y^{-}, y\right)} d P_{0}(\eta)}+\frac{t^{2}}{2}\left(P_{0}(y,+\infty)-P_{0}\left(-\infty, y^{-}\right)\right) \\
& <\frac{\int_{\left[y, y^{+}\right)}\left(\eta+t u_{0}(\eta)\right) d P_{0}(\eta)}{\int_{\left[y, y^{+}\right)} d P_{0}(\eta)}+\frac{t^{2}}{2}\left(P_{0}\left(y^{+}, \infty\right)-P_{0}(-\infty, y)\right) .
\end{aligned}
$$

Having $\left\{\xi_{t}\right\}_{t \geqq 0}$, we can construct $\varphi_{t}, u$ using (1.19), $P_{t}$ as before, and $I_{t}$ from $u$ and $P_{t}$ by a simple integration.

Definition. Let $\left(P_{t}, I_{t}\right)$ be a family of Borel measures, weakly continuous with respect to $t$, such that $I_{t}$ is absolutely continuous with respect to $P_{t}$ for each fixed $t$. Define $u$ via (1.16). $\left(P_{t}, I_{t}, u\right)_{t \geqq 0}$ is a weak solution of (1.2) iff for any 
$f, g \in C_{0}^{1}\left(R^{1}\right)$, and $0<t_{1}<t_{2}$,

$$
\int f(\eta) d P_{t_{2}}(\eta)-\int f(\eta) d P_{t_{1}}(\eta)=\int_{t_{1}}^{t_{2}} d \tau \int f^{\prime}(\eta) d I_{\tau}(\eta)
$$

$$
\begin{aligned}
\int g(\eta) d I_{t_{2}}(\eta)-\int g(\eta) d I_{t_{1}}(\eta)= & \int_{t_{1}}^{t_{2}} d \tau \int g^{\prime}(\eta) u(\eta, \tau) d I_{\tau}(\eta) \\
& +\int_{t_{1}}^{t_{2}} d \tau \int g(\eta)\left(P_{\tau}(\eta,-\infty)-P_{\tau}(-\infty, \eta)\right) d P_{\tau}(\eta)
\end{aligned}
$$

Theorem 2. Under the assumptions (A1-4), the family $\left(P_{t}, I_{t}, u\right)_{t \geqq 0}$ constructed using GVP gives a weak solution of (1.2) with initial data $\left(P_{0}, I_{0}\right)$ in the sense that

$$
\lim P_{t}=P_{0}, \quad \lim I_{t}=I_{0}
$$

weakly as $t \rightarrow 0^{+}$.

Before continuing, let us put these results in the perspective of general hyperbolic conservation laws. For obvious reasons, (1.1) is sometimes referred to as the pressureless gas dynamics equations. However, compared with the isentropic gas dynamics equation

$$
\left\{\begin{array}{r}
\rho_{t}+(\rho u)_{x}=0 \\
(\rho u)_{t}+\left(\rho u^{2}+p(\rho)\right)_{x}=0
\end{array}\right.
$$

there are two important differences. First at a technical level, the natural space for (1.1) is $M$, the space of Radon measures, instead of $B V$ or $L^{\infty}$. Secondly, the standard entropy condition, which in the present case, takes the form

$$
(\rho S(\rho))_{t}+(u \rho S(\rho))_{x} \leqq 0,
$$

where $S$ is convex, is not enough as a uniqueness criterion. Indeed in the context of particle systems, there is a whole family of inelastic collision rules that satisfy (1.1) and (1.23). The adhesion dynamics considered here is an extreme case of these collision rules. It is easy to see that the weak solutions of (1.1) constructed in this paper has the additional property:

$$
u_{x}<\frac{1}{t} \text {. }
$$

It is suggested by Jonathan Goodman that this might be sufficient as a uniqueness criterion. Moreover, it is natural to expect that the adhesion dynamics corresponds to a form of viscosity limit. But this also remains to be proven. Notice that (1.1) is an extreme case of a non-strictly hyperbolic system: the two characteristic fields coincide.

We will also consider a model closely related to (1.2) which is also discussed in the astrophysics literature [GMS, VDFN]:

$$
\left\{\begin{aligned}
\rho_{t}+(\rho u)_{x} & =0 \\
u_{t}+\left(\frac{u^{2}}{2}\right)_{x} & =-g_{x} \\
g_{x x} & =\rho .
\end{aligned}\right.
$$


The relation between (1.2) and (1.24) is analogous to the relation between (1.1) and (1.5-6). Let $h=g_{x}$, we can rewrite (1.24) as

$$
\left\{\begin{array}{c}
h_{t}+u h_{x}=0 \\
u_{t}+\left(\frac{u^{2}}{2}\right)_{x}=-h
\end{array}\right.
$$

If $P_{0}$ is absolutely continuous, we can reduce (1.25) to a scalar conservation law of the form

$$
u_{t}+\left(\frac{u^{2}}{2}\right)_{x}=-h_{0}(G(x-u t, t)),
$$

which is amenable to standard methods. Using this, we are able to establish both existence and uniqueness of global weak solutions for (1.24). This is explained in Sect. 6.

In the second part of this paper we study the qualitative behavior of these models with continuous, but non-differentiable initial data, extending some results from [S]. We show that the solutions of these models share the common feature that almost surely, at any $t>0$, all masses concentrate on points (i.e. the absolute continuous part vanishes), and the set of shock locations is dense. This behavior was to some extent anticipated by Zeldovich [Z] in his work on cosmology. In that context, these point masses are interpreted as the galaxies in a one-dimensional universe.

Before ending this introduction, let us mention that.(1.1) and (1.2) also have an origin in kinetic equations. Consider

$$
f_{t}+v f_{x}=0
$$

If we look for solutions of the form

$$
f(x, v, t)=\rho(x, t) \delta(v-u(x, t)),
$$

we obtain $(1.1)$ for $(\rho, u)$. Similarly consider the Vlasov-Poisson-Jeans equation [VDFN]

$$
\left\{\begin{array}{c}
f_{t}+v f_{x}-g_{x} f_{v}=0 \\
g_{x x}=\int f(x, v, t) d v
\end{array}\right.
$$

If we look for solutions of the form (1.28), we obtain (1.2) for $(\rho, u)$.

The paper has eight sections and one appendix. In Sect. 2, we compare our GVP with the Lax-Oleinik variational principle for scalar quasi-linear equations. In Sect. 3, we consider the discrete version of (1.1) and prove GVP for this case. In Sect. 4, we extend these results to the continuous case and complete the proof of Theorem 1. In Sect. 5, we explain the additional steps needed for the proof of Theorem 2. In Sect. 6, we consider the modified gravitational system (1.3).

Part II consists of two sections. In Sect.7, we consider (1.1) and (1.2) with random initial data. In Sect. 8, we extend these results to (1.3).

After this paper was submitted for publication, we received a preprint [BG] by Brenier and Grenier in which existence of weak solutions of (1.1) was proved without resorting to GVP at the continuous level. [BG] also contains some very interesting ideas for the multi-dimensional version of (1.1). We thank Brenier and Grenier for timely communication of their results. 


\section{Part I. Generalized Variational Principles and Global Weak Solutions}

\section{Preliminaries and Comparison with the Lax-Oleinik Variational Principle}

In the following we will concentrate on (1.1). The necessary changes for (1.2) will be summarized in Sect. 5 .

Intuition from adhesion dynamics suggests that the masses cluster more and more, and the accumulated masses will never split apart again. We formulate this as:

Lemma 1. The family of partitions $\left\{\xi_{t}\right\}_{t>0}$ determined with the help of GVP is decreasing. In other words, for $0<t^{\prime}<t$, each element of $\xi_{t}$ is contained in an element of $\xi_{t^{\prime}}$.

Proof. Assume to the contrary that there exists $y \in \partial \xi_{t}$, but $y \bar{\epsilon} \partial \xi_{t^{\prime}}$, where $\partial \xi_{t}$ denotes the collection of points belonging to the boundary of some element of $\xi_{t}$. Without loss of generality, we can assume that $y$ is the left end point of an element in $\xi_{t}$. Then for some $y^{-}<y<y^{+}$, we should have

$$
\frac{\int_{\left[y^{-}, y\right)}\left(\eta+t^{\prime} u_{0}(\eta)\right) d P_{0}(\eta)}{\int_{\left[y^{-}, y\right)} d P_{0}(\eta)} \geqq \frac{\int_{\left[y, y^{+}\right]}\left(\eta+t^{\prime} u_{0}(\eta)\right) d P_{0}(\eta)}{\int_{\left[y, y^{+}\right]} d P_{0}(\eta)} .
$$

Consider two linear functions $l_{1}(s), l_{2}(s)$ of $s$ defined by

$$
\begin{aligned}
& l_{1}(s)=\frac{\int_{\left[y^{-}, y\right)} \eta d P_{0}(\eta)}{\int_{\left[y^{-}, y\right)} d P_{0}(\eta)}+s \frac{\int_{\left[y^{-}, y\right)} u_{0}(\eta) d P_{0}(\eta)}{\int_{\left[y^{-}, y\right)} d P_{0}(\eta)}, \\
& l_{2}(s)=\frac{\int_{\left[y, y^{+}\right]} \eta d P_{0}(\eta)}{\int_{\left[y, y^{+}\right]} d P_{0}(\eta)}+s \frac{\int_{\left[y, y^{+}\right]} u_{0}(\eta) d P_{0}(\eta)}{\int_{\left[y, y^{+}\right]} d P_{0}(\eta)} .
\end{aligned}
$$

For sufficiently small $s$, we have $l_{1}(s)<l_{2}(s)$ while $l_{1}\left(t^{\prime}\right) \geqq l_{2}\left(t^{\prime}\right)$. Since $l_{1}$ and $l_{2}$ are linear, we conclude that $l_{1}(t)>l_{2}(t)$. This contradicts the fact that $y \in \partial \xi_{t}$ : i.e. $y$ satisfies GVP at time $t$.

Now we will compare GVP with the classical Lax-Oleinik variational principle see $[\mathrm{L}, \mathrm{O}]$. We assume that $P_{0}$ has a density $\rho_{0}$, and $0<$ const $\leqq \rho_{0}(x)<\infty$. Introduce

$$
\phi_{1}(y)=\int^{y}\left(\eta+t u_{0}(\eta)\right) \rho_{0}(\eta) d \eta, \quad \phi_{2}(y)=\int^{y} \rho_{0}(\eta) d \eta
$$

and

$$
c\left(y^{\prime}, y^{\prime \prime}\right)=\frac{\phi_{1}\left(y^{\prime \prime}\right)-\phi_{1}\left(y^{\prime}\right)}{\phi_{2}\left(y^{\prime \prime}\right)-\phi_{2}\left(y^{\prime}\right)}
$$


$\phi_{2}$ defines a $C^{1}$-diffeomorphism of $R^{1}$. Let $y=\phi_{2}^{-1}(z)$, and define

$$
\tilde{c}\left(z^{\prime}, z^{\prime \prime}\right)=\frac{\phi_{1} \circ \phi_{2}^{-1}\left(z^{\prime \prime}\right)-\phi_{1} \circ \phi_{2}^{-1}\left(z^{\prime}\right)}{z^{\prime \prime}-z^{\prime}} .
$$

In these notations, (1.14) becomes

$$
\inf _{y^{+}>y} c\left(y, y^{+}\right) \geqq \sup _{y^{-}<y} c\left(y^{-}, y\right)
$$

or, for $z=\phi_{2}(y)$

$$
\inf _{z^{+}>z} \tilde{c}\left(z, z^{+}\right) \geqq \sup _{z^{-}<z} \tilde{c}\left(z^{-}, z\right) .
$$

Let $\phi(z)=\phi_{1} \circ \phi_{2}^{-1}(z)$. Geometrically (2.7) means that $(z, \phi(z))$ is a point of contact of the graph of $\phi$ and its convex hull constructed in the coordinate $z$.

In comparison, the Lax-Oleinik variational principle for the Burgers equation (1.5) can be formulated as

$$
u(x, t)=\frac{\partial}{\partial x} \inf _{z}\left\{\frac{(x-z)^{2}}{2 t}+\int^{z} u_{0}(\eta) d \eta\right\} .
$$

It is easy to see that finding the minimum in (2.8) is the same as constructing the convex hull of the function $F(y)=\int^{y}\left(\eta+t u_{0}(\eta)\right) d \eta$ and finding the points of contact between the graphs of $F$ and its convex hull. In fact the set of points where the minimum in (2.8) is attained is exactly the same as the set of contact points between the graphs of $F$ and its convex hull. Notice that $F$ is a special case of $\phi_{1}$ when $\rho_{0} \equiv 1$. We see that the construction of GVP is the same as constructing the convex hull, but in a special coordinate $z$. It is in this sense that (1.14) and (1.21) generalize the variational principle of Lax and Oleinik.

\section{The Discrete Case}

It is instructive to consider first the case of a finite collection of particles $\left\{\left(x_{i}^{0}, v_{i}^{0}, m_{i}^{0}\right)\right.$, $i=1,2, \ldots, N\}$, where $x_{i}^{0}, v_{i}^{0}$ and $m_{i}^{0}$ are respectively the location, velocity, and mass of the $i^{\text {th }}$ particle. The particles undergo adhesion dynamics, defined in Sect. 1. The effect of the partitions $\left\{\xi_{t}\right\}_{t>0}$ is to divide the particles into ordered groups or clusters $G_{1}(t), G_{2}(t), \ldots, G_{k}(t)$, so that each group of particles are glued to a single one before or at time $t$, and different groups are at different locations at time $t$. If $G$ is one such group, say $G=\{i, i+1, \ldots, j\}$, we denote by $C_{G}(t)$ its location at time $t$. From the conservation of mass and momentum, we know that $C_{G}(t)$ has to be the center of mass of $G$ :

$$
C_{G}(t)=\frac{\sum_{l=i}^{j} m_{l}^{0}\left(x_{l}^{0}+t v_{l}^{0}\right)}{\sum_{l=i}^{j} m_{l}^{0}} .
$$

More generally, we will denote the expression on the right-hand side of (3.1) by $C_{i, j}(t)$. It is a linear function of $t$.

Lemma 2. Let $G_{1}$ and $G_{2}$ be two neighboring groups of particles such that $C_{G_{1}}(t)<C_{G_{2}}(t)$ for $t<t^{*}$, and $C_{G_{1}}\left(t^{*}\right)=C_{G_{2}}\left(t^{*}\right)$. Then for $t>t^{*}$,

$$
C_{G_{1} \cup G_{2}}(t)<C_{G_{1}}(t) .
$$


Proof. Since both $C_{G_{1}}(t)$ and $C_{G_{2}}(t)$ are linear functions of $t$, we have for $t>t^{*}$,

$$
C_{G_{1}}(t)>C_{G_{2}}(t) .
$$

Since $C_{G_{1} \cup G_{2}}(t)=\alpha C_{G_{1}}(t)+(1-\alpha) C_{G_{2}}(t)$ for some constant $\alpha \in(0,1)$, we have for $t>t^{*}$,

$$
C_{G_{1} \cup G_{2}}(t)<C_{G_{1}}(t)
$$

This proves the lemma.

Lemma 3. Let $G \in \xi_{t}$, and $G=\left\{x_{i}^{0}, j^{\prime} \leqq i \leqq j^{\prime \prime}\right\}$. Then for any $i, j^{\prime} \leqq i \leqq$ $j^{\prime \prime}-1$, we have

$$
C_{j^{\prime}, i}(t) \geqq C_{i+1, j^{\prime \prime}}(t) .
$$

Proof. Assume to the contrary that there exists an $i$, such that (3.2) does not hold. Since $C_{j^{\prime}, j^{\prime \prime}}(t)=\alpha C_{j^{\prime}, i}(t)+(1-\alpha) C_{i+1, j^{\prime \prime}}(t)$ for some $0 \leqq \alpha \leqq 1$, we have:

$$
C_{j^{\prime}, i}(t)<C_{j^{\prime}, j^{\prime \prime}}(t)
$$

Consider the evolution of the set of particles $I(0)=\left\{x_{j}^{0}, j^{\prime} \leqq j \leqq i\right\}$. Each time the set is hit from the right by a particle or a cluster of particles, we add them to our set. In this way we obtain a growing family of sets $I(s)=\left\{x_{j}^{0}, j^{\prime} \leqq j \leqq i(s)\right\}$. From Lemma 2, when new particles are added to $I(s)$, its center of mass is moved further to the left, i.e.

$$
C_{j^{\prime}, i(s)}(s)<C_{j^{\prime}, i}(s) .
$$

From the assumption of the lemma we have $i(t)=j^{\prime \prime}$. Hence we have

$$
C_{j^{\prime}, j^{\prime \prime}}(t)<C_{j^{\prime}, i}(t)
$$

contradicting (3.3).

Theorem 1'. $x_{j}^{0}$ is the left endpoint of an element of the partition $\xi_{t}$ iff

$$
\max _{j^{\prime}<j} C_{j^{\prime}, j-1}(t)<\min _{j^{\prime \prime} \geqq j} C_{j, j^{\prime \prime}}(t) .
$$

Proof. Assume that (3.6) holds, and $x_{j}^{0}$ is not the left endpoint of an element of $\xi_{t}$. Let $G \in \xi_{t}$ be the element of $\xi_{t}$ containing $x_{j}^{0}, G=\left\{x_{i}^{0}, i_{0} \leqq i \leqq j_{0}\right\}$ and $i_{0}<j$. From Lemma 3, we have

$$
C_{i_{0}, j-1}(t) \geqq C_{j, j_{0}}(t) .
$$

This clearly contradicts (3.6).

Assume now that $x_{j}^{0}$ is the left end point of an element of $\xi_{t}$. For any $j^{\prime}, j^{\prime \prime}, j^{\prime}<j<j^{\prime \prime}$, we want to show that $C_{j^{\prime}, j-1}(t)<C_{j, j^{\prime \prime}}(t)$. Let $I_{1}, I_{2}, \ldots, I_{l}$ be the consecutive elements of $\xi_{t}$ to the left of $x_{j}^{0}$, and $x_{j^{\prime}}^{0} \in I_{1}=\left\{x_{i}^{0}, i_{1} \leqq i \leqq i_{2}\right\}$. Let $J_{1}, J_{2}, \ldots, J_{r}$ be the consecutive elements of $\xi_{t}$ to the right of $x_{j}^{0}$ (including the one containing $x_{j}^{0}$ ), and $x_{j^{\prime \prime}}^{0} \in J_{r}=\left\{x_{i}^{0}, j_{1} \leqq i \leqq j_{2}\right\}$. From Lemma 3 , we have

$$
C_{j^{\prime}, i_{2}}(t) \leqq C_{I_{1}}(t), \quad C_{j_{1}, j^{\prime \prime}}(t) \geqq C_{I_{2}}(t) .
$$

We also have

$$
C_{I_{1}}(t)<\cdots<C_{I_{l}}(t)<C_{J_{1}}(t)<\cdots<C_{J_{r}}(t)
$$


Hence, we must have

$$
C_{j^{\prime}, j-1}(t)<C_{j, j^{\prime \prime}}(t) .
$$

We now show that the adhesion dynamics gives rise to weak solutions of (1.1) with initial data $P_{0}=\sum_{i=1}^{N} m_{i}^{0} \delta\left(x-x_{i}^{0}\right), I_{0}=\sum_{i=1}^{N} m_{i}^{0} v_{i}^{0} \delta\left(x-x_{i}^{0}\right)$.

Let $\left\{x_{j}(t+\Delta t), v_{j}(t+\Delta t), m_{j}(t+\Delta t), j=1, \ldots, N(t)\right\}$ be the locations, velocities, and masses of the particles at time $t+\Delta t$. Assume that the $j^{\text {th }}$ particle is formed by gluing together some particles whose locations, velocities, and masses at time $t$ were respectively $x_{i j}(t), v_{i j}(t)$ and $m_{i j}(t), i \in I_{j}$-a set of indices depending on $j$. We have

$$
\begin{aligned}
x_{j}(t+\Delta t) & =\frac{\sum_{i \in I_{j}}\left(x_{i j}(t)+\Delta t v_{i j}(t)\right) m_{i j}(t)}{m_{j}(t+\Delta t)}, \\
v_{j}(t+\Delta t) & =\frac{\sum_{i \in I_{j}} v_{i j}(t) m_{i j}(t)}{m_{j}(t+\Delta t)}, \\
m_{j}(t+\Delta t) & =\sum_{i \in I_{j}} m_{i j}(t) .
\end{aligned}
$$

We also have $\left|x_{i j}(t)-x_{j}(t+\Delta t)\right| \leqq$ Const $\cdot \Delta t$ since all velocities are bounded. Let $f, g \in C_{0}^{1}\left(R^{1}\right)$. We first show that $\int f(\eta) d P_{t}(\eta)$ is a differentiable function of $t$, and

$$
\frac{d}{d t} \int f(\eta) d P_{t}(\eta)=\int f^{\prime}(\eta) d I_{t}(\eta)
$$

This implies (D1). Indeed, we have

$$
\begin{aligned}
\int f(\eta) d P_{t}(\eta)= & \sum_{i, j} f\left(x_{i j}(t)\right) m_{i j}(t) \\
= & \sum_{j} \sum_{i \in I_{j}} f\left(x_{j}(t+\Delta t)+x_{i j}(t)-x_{j}(t+\Delta t)\right) m_{i j}(t) \\
= & \sum_{j} f\left(x_{j}(t+\Delta t)\right) m_{j}(t+\Delta t) \\
& +\sum_{j} f^{\prime}\left(x_{j}(t+\Delta t)\right) \sum_{i \in I_{j}}\left(x_{i j}(t)-x_{j}(t+\Delta t)\right) m_{i j}(t)+o(\Delta t) .
\end{aligned}
$$

Since

$$
\begin{aligned}
\sum_{i \in I_{j}}\left(x_{i j}(t)-x_{j}(t+\Delta t)\right) m_{i j}(t) & =\sum_{i} x_{i j}(t) m_{i j}(t)-x_{j}(t+\Delta t) m_{j}(t+\Delta t) \\
& =-\Delta t \sum_{i} v_{i j}(t) m_{i j}(t)=-\Delta t v_{j}(t+\Delta t) m_{j}(t+\Delta t)
\end{aligned}
$$

we obtain

$$
\begin{aligned}
& \int f(\eta) d P_{t}(\eta) \\
& \quad=\int f(\eta) d P_{t+\Delta t}(\eta)-\Delta t \sum_{j} f^{\prime}\left(x_{j}(t+\Delta t)\right) v_{j}(t+\Delta t) m_{j}(t+\Delta t)+o(\Delta t) .
\end{aligned}
$$

This implies (3.12). 
In contrast $\int g(\eta) d I_{t}(\eta)$ is not $C^{1}$ in $t$ because of the inelastic nature of the collisions. To see this, consider just two particles colliding at $t=\tau$. Before collision they are denoted by $\left(x_{1}(t), v_{1}(t), m_{1}(t)\right),\left(x_{2}(t), v_{2}(t), m_{2}(t)\right), t<\tau$, respectively. After collision they form a single particle $(x(t), v(t), m(t)), t \geqq \tau$. We have $m(\tau)=m_{1}(\tau)+m_{2}(\tau), \quad m(\tau) v(\tau)=m_{1}(\tau) v_{1}(\tau)+m_{2}(\tau) v_{2}(\tau), \quad m(\tau) x(\tau)=$ $m_{1}(\tau) x_{1}(\tau)+m_{2}(\tau) x_{2}(\tau)$. For $t<\tau$, we have

$$
\begin{aligned}
\int g(\eta) d I_{t}(\eta)= & g\left(x_{1}(t)\right) v_{1}(t) m_{1}(t)+g\left(x_{2}(t)\right) v_{2}(t) m_{2}(t) \\
= & g(x(\tau))\left(v_{1}(t) m_{1}(t)+v_{2}(t) m_{2}(t)\right) \\
& +(t-\tau) g^{\prime}(x(\tau))\left(v_{1}^{2}(t) m_{1}(t)+v_{2}^{2}(t) m_{2}(t)\right)+O\left((t-\tau)^{2}\right) \\
= & \int g(\eta) d I_{\tau}(\eta)+(t-\tau) g^{\prime}(x(\tau))\left(v_{1}^{2}(\tau) m_{1}(\tau)+v_{2}^{2}(\tau) m_{2}(\tau)\right) \\
& +O\left((t-\tau)^{2}\right) .
\end{aligned}
$$

For $t>\tau$, we have

$$
\begin{aligned}
\int g(\eta) d I_{t}(\eta) & =g(x(t)) v(t) m(t) \\
& =\int g(\eta) d I_{\tau}(\eta)+(t-\tau) g^{\prime}(x(\tau)) v^{2}(\tau) m(\tau)+O\left((t-\tau)^{2}\right) .
\end{aligned}
$$

In general, energy decreases at collisions

$$
v_{1}^{2}(\tau) m_{1}(\tau)+v_{2}^{2}(\tau) m_{2}(\tau) \neq v^{2}(\tau) m(\tau) .
$$

Hence $\int g(\eta) d I_{t}(\eta)$ is not $C^{1}$ in $t$. Nevertheless, we can prove that (D2) is still valid. We only have to prove this for the case when there is no collision in $\left(t_{1}, t_{2}\right)$ and at $t=t_{2}$, a group of particles, say with indices $i_{1}, i_{1}+1, \ldots, i_{2}$, are colliding to form a single particle at $x\left(t_{2}\right)$. We then have

$$
\begin{aligned}
\int_{t_{1}}^{t_{2}} d \tau \int g^{\prime}(\eta) u(\eta, \tau) d I_{\tau}(\eta)=\int_{t_{1}}^{t_{2}} d \tau \sum_{i=1}^{N} g^{\prime}\left(x_{i}(\tau)\right) v_{i}^{2}(\tau) m_{i} \\
=\int_{t_{1}}^{t_{2}} d \tau\left[\sum_{i=1}^{i_{1}-1} m_{i} v_{i} \frac{d}{d \tau} g\left(x_{i}(\tau)\right)+\sum_{i=i_{1}}^{i_{2}} m_{i} v_{i} \frac{d}{d \tau} g\left(x_{i}(\tau)\right)+\sum_{i=i_{2}+1}^{N} m_{i} v_{i} \frac{d}{d \tau} g\left(x_{i}(\tau)\right)\right] \\
=\sum_{i=1}^{i_{1}-1} m_{i} v_{i} g\left(x_{i}\left(t_{2}\right)\right)+g\left(x\left(t_{2}\right)\right) \sum_{i=i_{1}}^{i_{2}} m_{i} v_{i}+\sum_{i=i_{2}+1}^{N} m_{i} v_{i} g\left(x_{i}\left(t_{2}\right)\right)-\sum_{i=1}^{N} m_{i} v_{i} g\left(x_{i}\left(t_{1}\right)\right) \\
=\int g(\eta) d I_{t_{2}}(\eta)-\int g(\eta) I_{t_{1}}(\eta) .
\end{aligned}
$$

\section{Proof of Theorem 1}

We will first give the proof for the case when $P_{0}$ is absolutely continuous, and then for the case when $P_{0}$ is discrete.

For initial data satisfying (A1-A3), we construct a decreasing family of partitions $\left\{\xi_{t}\right\}_{t>0}$ of $R^{1}$ according to GVP (1.14). Having $\left\{\xi_{t}\right\}_{t>0}$, we can define $\varphi_{t}, P_{t}, I_{t}$, and $u(x, t)$. Obviously $\varphi_{t}$ is a non-decreasing function of $y$ for any fixed $t$. 
Furthermore, as a consequence of the assumption that $u(x ; 0)$ is continuous, $\varphi_{t}$ is also continuous, and we have

$$
\begin{aligned}
\int f(\eta) d P_{t}(\eta) & =\int f\left(\varphi_{t}(\eta)\right) d P_{0}(\eta), \\
\int g(\eta) d I_{t}(\eta) & =\int g\left(\varphi_{t}(\eta)\right) d I_{0}(\eta) .
\end{aligned}
$$

We will prove Theorem 1 via discrete approximations. Take a sequence of measures $P_{0}^{(n)}$ concentrated on finite sets $\left\{x_{i}^{(n)}, i=1, \ldots,\right\}$ such that $P_{0}^{(n)} \rightarrow P_{0}$ weakly. Define $I_{0}^{(n)}$ to be a signed measure concentrated on the set $\left\{x_{i}^{(n)}, i=1, \ldots,\right\}$ such that $I_{0}^{(n)}\left(\left\{x_{i}^{(n)}\right\}\right)=u_{0}\left(x_{i}^{(n)}\right) P_{0}^{(n)}\left(\left\{x_{i}^{(n)}\right\}\right)$. Then $I_{0}^{(n)} \rightarrow I_{0}$ weakly. Using GVP, we construct the corresponding families of partitions $\xi_{t}^{(n)}$ and mappings $\varphi_{t}^{(n)}$. Moreover we already showed (Theorem $1^{\prime}$ ) that for $f, g \in C_{0}\left(R^{1}\right)$,

$$
\begin{aligned}
\int f(\eta) d P_{t}^{(n)} & =\int f\left(\varphi_{t}^{(n)}(\eta)\right) d P_{0}^{(n)}, \\
\int g(\eta) d I_{t}^{(n)} & =\int g\left(\varphi_{t}^{(n)}(\eta)\right) d I_{0}^{(n)}
\end{aligned}
$$

Here $P_{t}^{(n)}, I_{t}^{(n)}$ are constructed in Sect. 1. We also have for $0<t_{1}<t_{2}$,

$$
\begin{aligned}
\int f(\eta) d P_{t_{2}}^{(n)}-\int f(\eta) d P_{t_{1}}^{(n)} & =\int_{t_{1}}^{t_{2}} d \tau \int f^{\prime}(\eta) d I_{\tau}^{(n)}=\int_{t_{1}}^{t_{2}} d \tau \int f^{\prime}\left(\varphi_{\tau}^{(n)}(\eta)\right) d I_{0}^{(n)}, \\
\int g(\eta) d I_{t_{2}}^{(n)}-\int g(\eta) d I_{t_{1}}^{(n)} & =\int_{t_{1}}^{t_{2}} d \tau \int g^{\prime}\left(\varphi_{\tau}^{(n)}(\eta)\right) u^{(n)}(\eta, \tau) d I_{0}^{(n)}
\end{aligned}
$$

We can extend the definition of $\varphi_{t}^{(n)}$ to the whole line by putting $\varphi_{t}^{(n)}(y)=\varphi_{t}^{(n)}\left(x_{i}^{(n)}\right)$ if $x_{i}^{(n)} \leqq y \leqq x_{i+1}^{(n)}, \varphi_{t}^{(n)}(y)=\varphi_{t}^{(n)}\left(x_{1}^{(n)}\right)$ if $y<x_{1}^{(n)}, \varphi_{t}^{(n)}(y)=\varphi_{t}^{(n)}\left(x_{N}^{(n)}\right)$ if $y \geqq$ $x_{N}^{(n)}$. Here $x_{1}^{(n)}=\min \left\{x_{i}^{(n)}\right\}, x_{N}^{(n)}=\max \left\{x_{i}^{(n)}\right\}$.

We will use superscript " $n$ " to denote objects corresponding to $P_{0}^{(n)}, I_{0}^{(n)}$. If $\Delta$ is an interval in $R^{1}$, we denote

$$
C_{\Delta}(t)=\frac{\int_{\Delta}(\eta+t u(\eta ; 0)) d P_{0}(\eta)}{\int_{\Delta} d P_{0}(\eta)}, \quad u_{0}(\Delta)=\frac{\int_{\Delta} u(\eta ; 0) d P_{0}(\eta)}{\int_{\Delta} d P_{0}(\eta)}
$$

The following lemma is a continuous version of Lemma 3. It can be proven in the same way as Lemma 3.

Lemma $3^{\prime}$. Let $G=[a, b]$ be an element of $\xi_{t}, a<b$. Then for any $c \in(a, b)$, we have

$$
C_{[a, c)}(t) \geqq C_{[c, b]}(t) .
$$

Lemma 4. $\varphi^{(n)} \rightarrow \varphi$ uniformly on compact subsets of $R^{1} \times[0, \infty)$.

Proof. We first prove that for any fixed $t \geqq 0$, the inverse images of $\varphi_{t}^{(n)}$ of a bounded interval $L=(c, d)$ are uniformly bounded. To that end, let $A^{(n)}=$ $\left\{x_{i}^{(n)}, \varphi_{t}^{(n)}\left(x_{i}^{(n)}\right) \in L\right\}$, and $x_{\min }^{(n)}=\min \left\{x_{i}^{(n)}, x_{i}^{(n)} \in A^{(n)}\right\}$. Obviously $x_{\min }^{(n)}$ has to be the left end point of an element $G^{(n)}$ in the partition $\xi_{t}^{(n)}$. From Lemma 3, we have

$$
x_{\min }^{(n)}+t u_{0}\left(x_{\min }^{(n)}\right) \geqq C_{G^{(n)}}^{(n)}(t) \geqq c .
$$


Using (A3), we can write (4.6) as

$$
x_{\min }^{(n)}(1+o(1) t) \geqq c .
$$

Hence $\left\{x_{\min }^{(n)}\right\}$ is uniformly bounded from below. Similarly we can prove an upper bound.

Assume to the contrary that $\left\{\varphi_{t}^{(n)}\right\}$ do not converge uniformly on some bounded set, say $[c, d] \times[0, T]$. Then there exists $\varepsilon>0$, and sequences $\left\{y_{n}, t_{n}\right\} \in[c, d] \times$ $[0, T]$ such that

$$
\left|\varphi_{t_{n}}^{(n)}\left(y_{n}\right)-\varphi_{t_{n}}\left(y_{n}\right)\right|>\varepsilon
$$

We can choose $\left\{y_{n}\right\},\left\{t_{n}\right\}$ such that $\lim y_{n}=y^{*}, \lim t_{n}=t, \lim \varphi_{t}^{(n)}\left(y_{n}\right)=x^{*}$ exist. Since

$$
\left|\varphi_{t_{n}}^{(n)}\left(y_{n}\right)-\varphi_{t}^{(n)}\left(y_{n}\right)\right| \leqq\left|t_{n}-t\right| \max |u(x ; 0)| \leqq \text { Const }\left|t_{n}-t\right|
$$

where the maximum is taken over a bounded set, for sufficiently large $n$ we get from $(4.8)$

$$
\left|\varphi_{t}^{(n)}\left(y_{n}\right)-\varphi_{t}\left(y_{n}\right)\right|>\frac{\varepsilon}{2}
$$

Therefore we have

$$
\left|x^{*}-\varphi_{t}\left(y^{*}\right)\right| \geqq \frac{\varepsilon}{2} .
$$

Without loss of generality, let us assume $x^{*}<\varphi_{t}\left(y^{*}\right)$. Let $\Delta_{n}=\left(\varphi_{t}^{(n)}\right)^{-1}$ $\left\{\varphi_{t}^{(n)}\left(y_{n}\right)\right\}=\left\{x_{i_{n}}^{(n)}<\cdots<x_{j_{n}}^{(n)}\right\}$ which is an element of $\xi_{t}^{(n)}$. By extracting subsequences, we can assume that $\Delta_{n}$ converges to an interval $\Delta$, with $\bar{\Delta}=[a, b]$, in the sense that

$$
\lim x_{i_{n}}^{(n)}=a, \quad \lim x_{j_{n}}^{(n)}=b .
$$

Consequently, we also have $x^{*}=C_{\Delta}(t) . \Delta$ itself may be open, closed, or half-open. Since $y_{n} \in \Delta_{n}$, we must have $y^{*} \in \bar{\Delta}$. Let $\varphi_{t}^{-1}\left\{\varphi_{t}\left(y^{*}\right)\right\}=[c, d]$. By the continuity of $\varphi_{t}$, this has to be a closed interval which might consist of a single point. We will discuss the case when $a<b$. The reader can easily see that the case when $a=b$ also follows.

First we prove that $c, d \bar{\epsilon}(a, b)$. Assume to the contrary that $c \in(a, b)$. We can choose a sequence $\left\{x_{l_{n}}^{(n)}\right\}, x_{i_{n}}^{(n)}<x_{l_{n}}^{(n)}<x_{j_{n}}^{(n)}$, such that $\lim x_{l_{n}}^{(n)}=c$. Since $x_{l_{n}}^{(n)}$ is not the end point of any element in $\xi_{t}^{(n)}$, there exist $j_{n}^{\prime}, j_{n}^{\prime \prime}$, such that

$$
C_{j_{n}^{\prime}, l_{n-1}}^{(n)}(t) \geqq C_{l_{n}, j_{n}^{\prime \prime}}^{(n)}(t) .
$$

By extracting subsequences, we can further assume that $\left\{x_{j_{n}^{\prime}}^{(n)}\right\}$ and $\left\{x_{j_{n}^{\prime \prime}}^{(n)}\right\}$ converge to $c^{*}$ and $d^{*}$, respectively.

Taking the limit of (4.13), we obtain

$$
\frac{\int_{c^{*}}^{c}\left(\eta+t u_{0}(\eta)\right) d P_{0}(\eta)}{\int_{c^{*}}^{c} d P_{0}(\eta)} \geqq \frac{\int_{c}^{d^{*}}\left(\eta+t u_{0}(\eta)\right) d P_{0}(\eta)}{\int_{c}^{d^{*}} d P_{0}(\eta)}
$$

contradicting (1.14). Therefore $c \bar{\epsilon}(a, b)$. Similarly $d \bar{\in}(a, b)$. 
We are left with three different cases.

Case 1. $a<b=y^{*}=c<d$. From

$$
C_{i_{n}, j_{n}}^{(n)}(t) \geqq C_{j_{n}, j_{n}}^{(n)}(t)
$$

a limiting argument similar to the one presented above gives

$$
x^{*} \geqq y^{*}+t u_{0}\left(y^{*}\right) .
$$

On the other hand, from Lemma $3^{\prime}$, we have

$$
y^{*}+t u_{0}\left(y^{*}\right) \geqq \varphi_{t}\left(y^{*}\right) .
$$

Hence we have $x^{*} \geqq \varphi_{t}\left(y^{*}\right)$ contradicting to the assumption that $x^{*}<\varphi_{t}\left(y^{*}\right)$.

Case 2. $c<a<b<d$. Choosing $\left\{x_{l_{n}}^{(n)}\right\}$ monotonically decreasing to $c$, and using $C_{l_{n}, i_{n}-1}^{(n)}(t)<\varphi_{t}^{(n)}\left(y_{n}\right)$ we obtain

$$
C_{[c, a]}(t) \leqq x^{*}
$$

From Lemma $3^{\prime}$ we get

$$
\varphi_{t}\left(y^{*}\right) \leqq C_{[c, a]}(t)
$$

Again we get $x^{*} \geqq \varphi_{t}\left(y^{*}\right)$.

Case 3. $c<d=y^{*}=a<b$. The proof of Case 2 applies to this case also.

Proof of Theorem 1. To complete the proof of Theorem 1, we have to establish (D1) and (D2).

For any $t>0$, we write

$$
\begin{aligned}
\int f(\eta) d P_{t}(\eta)= & \int\left[f\left(\varphi_{t}(\eta)\right)-f\left(\varphi_{t}^{(n)}(\eta)\right)\right] d P_{0}^{(n)}(\eta)+\int f\left(\varphi_{t}(\eta)\right)\left(d P_{0}(\eta)\right. \\
& \left.-d P_{0}^{(n)}(\eta)\right)+\int f\left(\varphi_{t}^{(n)}(\eta)\right) d P_{0}^{(n)}(\eta) \\
= & \int f\left(\varphi_{t}^{(n)}(\eta)\right) d P_{0}^{(n)}(\eta)+\delta_{t, n}^{(1)}+\delta_{t, n}^{(2)}
\end{aligned}
$$

where $\delta_{t, n}^{(1)}, \delta_{t, n}^{(2)} \rightarrow 0$ as $n \rightarrow \infty$. Similarly we also have

$$
\begin{aligned}
& \int_{t_{1}}^{t_{2}} d t \int f^{\prime}(\eta) d I_{t}(\eta)=\int_{t_{1}}^{t_{2}} d t \int f^{\prime}\left(\varphi_{t}(\eta)\right) d I_{0}(\eta) \\
& =\int_{t_{1}}^{t_{2}} d t \int\left[f^{\prime}\left(\varphi_{t}(\eta)\right)-f^{\prime}\left(\varphi_{t}^{(n)}(\eta)\right)\right] d I_{0}^{(n)}(\eta)+\int_{t_{1}}^{t_{2}} d t \int f^{\prime}\left(\varphi_{t}(\eta)\right)\left[d I_{0}(\eta)\right. \\
& \left.\quad-d I_{0}^{(n)}(\eta)\right]+\int_{t_{1}}^{t_{2}} d t \int f^{\prime}\left(\varphi_{t}^{(n)}(\eta)\right) d I_{0}^{(n)}(\eta) \\
& =\int_{t_{1}}^{t_{2}} d t \int f^{\prime}\left(\varphi_{t}^{(n)}(\eta)\right) d I_{0}^{(n)}(\eta)+\beta_{t, n}^{(1)}+\beta_{t, n}^{(2)},
\end{aligned}
$$

where $\beta_{t, n}^{(1)}, \beta_{t, n}^{(2)} \rightarrow 0$ as $n \rightarrow \infty$. Together with the results of Sect. 3, we obtain (D1). 
To get (D2), let

$$
\begin{aligned}
\omega(y, t) & =\frac{\int_{C_{t}(y)} u_{0}(\eta) d P_{0}(\eta)}{\int_{C_{t}(y)} d P_{0}(\eta)}=u_{0}\left(C_{t}(y)\right), \\
\omega^{(n)}(y, t) & =\frac{\int_{C_{t}^{(n)}(y)} u_{0}^{(n)}(\eta) d P_{0}^{(n)}(\eta)}{\int_{C_{t}^{(n)}(y)} d P_{0}^{(n)}(\eta)}=u_{0}^{(n)}\left(C_{t}^{(n)}(y)\right) .
\end{aligned}
$$

The difficulty lies in the fact that $\omega^{(n)}(y, t)$ may not converge to $\omega(y, t)$. Roughly speaking, at the point of shock interactions, $C_{t}(y)$ may differ from $C_{t}^{(n)}(y)$ by a set of finite measure. The main remaining task, therefore, is to show that such interactions occur rather rarely and give a small contribution to the integrals in (D2).

Let $\delta>0$. By continuity of $u(\cdot, 0)$, we can choose $\varepsilon=\varepsilon(\delta)>0$, such that if $\left|C_{t}(y) \Delta C_{t}^{(n)}(y)\right|<\varepsilon$ (where $\Delta$ denotes symmetric difference), then

$$
\left|\omega(y, t)-\omega^{(n)}(y, t)\right|<\delta .
$$

Let $(y, \tau)$ be such that

$$
\left|\omega(y, \tau)-\omega^{(n)}(y, \tau)\right|>\delta,
$$

and let $C_{\tau}(y)=[A, B], C_{\tau}^{(n)}(y)=[C, D]$. Without loss of generality, we assume $A<C<B<D$. Then either, $D-B>\varepsilon$, or $C-A>\varepsilon$.

Let us assume that $D-B>\varepsilon$, and denote $t=\tau+\gamma, \varphi_{t}^{-1}\left(\varphi_{t}(y)\right)=\left[A^{\prime}, B^{\prime}\right]$. Assume $B^{\prime}<D$, we want to estimate $\gamma$, i.e. the time needed for the atom at $\varphi_{\tau}(y)$ to absorb half of the masses in $(B, D)$. We have

$$
\begin{aligned}
C_{\left[B^{\prime}, D\right]}(t) & =C_{\left[B^{\prime}, D\right]}(\tau)+\gamma u_{0}\left(\left[B^{\prime}, D\right]\right)=C_{\left[B^{\prime}, D\right]}^{(n)}(\tau)+\gamma u_{0}\left(\left[B^{\prime}, D\right]\right)+o(1) \\
& \leqq \varphi_{\tau}^{(n)}(y)+\gamma u_{0}\left(\left[B^{\prime}, D\right]\right)+o(1) .
\end{aligned}
$$

Here $o(1)$ denotes quantities that converge to 0 as $n$ goes to infinity, with all the other parameters such as $\varepsilon, \delta$ held fixed. Since the center of mass is a linear function of $t$, and

$$
C_{[A, B]}(\tau) \geqq C_{[C, B]}(\tau)
$$

we also have

$$
\begin{aligned}
C_{\left[A, B^{\prime}\right]}(t) \geqq C_{\left[C, B^{\prime}\right]}(t) & =C_{\left[C, B^{\prime}\right]}^{(n)}(\tau)+\gamma u_{0}\left(\left[C, B^{\prime}\right]\right)+o(1) \\
& \geqq \varphi_{\tau}^{(n)}\left(y^{*}\right)+\gamma u_{0}\left(\left[C, B^{\prime}\right]\right)+o(1) .
\end{aligned}
$$

From the definition of $B^{\prime}$, we have

$$
C_{\left[A, B^{\prime}\right]}(t) \leqq C_{\left[A^{\prime}, B^{\prime}\right]}(t)<C_{\left[B^{\prime}, D\right]}(t) .
$$

Therefore, we get

$$
\gamma\left\{u_{0}\left(\left[C, B^{\prime}\right]\right)-u_{0}\left(\left[B^{\prime}, D\right]\right)\right\} \leqq o(1) .
$$

On the other hand, since $C_{\left[C, B^{\prime}\right]}^{(n)}(\tau) \geqq C_{\left[B^{\prime}, D\right]}^{(n)}(\tau)$, we have

$$
C_{\left[C, B^{\prime}\right]}(\tau) \geqq C_{\left[B^{\prime}, D\right]}(\tau)+o(1) .
$$


Hence, we get

$$
\begin{aligned}
\tau\left\{u_{0}\left(\left[C, B^{\prime}\right]\right)-u_{0}\left(\left[B^{\prime}, D\right]\right)\right\} & \geqq \frac{\int_{B^{\prime}}^{D} \eta d P_{0}(\eta)}{\int_{B^{\prime}}^{D} d P_{0}(\eta)}-\frac{\int_{C}^{B^{\prime}} \eta d P_{0}(\eta)}{\int_{C}^{B^{\prime}} d P_{0}(\eta)}+o(1) \\
& \geqq \beta(\varepsilon)+o(1) .
\end{aligned}
$$

Here $\beta(\varepsilon)$ is some finite quantity depending on $\varepsilon$. Hence, we have

$$
\gamma \leqq \frac{o(1)}{\beta(\varepsilon)} \text {. }
$$

Similarly, if $C-A>\varepsilon$, we can show that after time $\frac{o(1)}{\beta(\varepsilon)}$, at least half of the masses in $(A, C)$ are absorbed by the atom at $\varphi_{\tau}^{(n)}(y)$.

Let $S=\left\{\tau \in\left[t_{1}, t_{2}\right]\right.$, there exists $y \in L, L$ is a sufficiently large interval, such that (4.19) holds $\}$. Since either $P_{0}(A, C) \geqq$ Const $\cdot \varepsilon$, or $P_{0}(B, D) \geqq$ Const $\cdot \varepsilon$, and since $P_{0}(L)<$ Const, we must have

$$
|S|<\frac{o(1)}{\beta(\varepsilon) \varepsilon} .
$$

Now let $g \in C_{0}^{1}\left(R^{1}\right)$. We choose $L$ to contain the support of $g\left(\varphi_{\tau}(\cdot)\right)$, $\tau \in\left[t_{1}, t_{2}\right]$,

$$
\begin{aligned}
& \left|\int_{t_{1}}^{t_{2}} d \tau \int g^{\prime}(\eta) u_{\tau}^{(n)}(\eta)^{2} d P_{\tau}^{(n)}(\eta)-\int_{t_{1}}^{t_{2}} d \tau \int g^{\prime}(\eta) u(\eta, \tau)^{2} d P_{\tau}(\eta)\right| \\
& =\left|\int_{t_{1}}^{t_{2}} d \tau \int g^{\prime}\left(\varphi_{\tau}^{(n)}(\eta)\right) \omega^{(n)}(\eta, \tau)^{2} d P_{0}^{(n)}(\eta)-\int_{t_{1}}^{t_{2}} d \tau \int g^{\prime}\left(\varphi_{\tau}(\eta)\right) \omega(y, \tau)^{2} d P_{0}(\eta)\right| \\
& \leqq\left|\int_{t_{1}}^{t_{2}} d \tau \int\left(g^{\prime}\left(\varphi_{\tau}^{(n)}(\eta)\right)-g^{\prime}\left(\varphi_{\tau}(\eta)\right)\right) \omega^{(n)}(\eta, \tau)^{2} d P_{0}^{(n)}(\eta)\right| \\
& +\left|\int_{t_{1}}^{t_{2}} d \tau \int g^{\prime}\left(\varphi_{\tau}(\eta)\right)\left[\omega^{(n)}(\eta, \tau)^{2}-\omega(\eta, \tau)^{2}\right] d P_{0}(\eta)\right| \\
& \leqq o(1)+\left|\int_{S} d \tau \int g^{\prime}\left(\varphi_{\tau}(\eta)\right)\left[\omega^{(n)}(\eta, \tau)^{2}-\omega(\eta, \tau)^{2}\right] d P_{0}(\eta)\right| \\
& +\left|\int_{\left[t_{1}, t_{2}\right] \backslash S} d \tau \int g^{\prime}\left(\varphi_{\tau}(\eta)\right)\left[\omega^{(n)}(\eta, \tau)^{2}-\omega(\eta, \tau)^{2}\right] d P_{0}(\eta)\right| \\
& \leqq o(1)+\text { Const } \cdot|S|+\text { Const } \cdot \delta \leqq o(1)+\frac{o(1)}{\beta(\varepsilon) \varepsilon}+\text { Const } \cdot \delta,
\end{aligned}
$$

where the constants depend on the support of $g$. Now we can choose $n$ so large that $\frac{o(1)}{\beta(\varepsilon) \varepsilon}+o(1)<\delta$. Then we get

$$
\left|\int_{t_{1}}^{t_{2}} d \tau \int g^{\prime}(\eta) u_{\tau}^{(n)}(\eta)^{2} d P_{\tau}^{(n)}(\eta)-\int_{t_{1}}^{t_{2}} d \tau \int g^{\prime}(\eta) u(\eta, \tau)^{2} d P_{\tau}(\eta)\right| \leqq \text { Const } \cdot \delta .
$$

Together with the argument which proved (D1), we get (D2). 
Now we discuss in detail the case of discrete $P_{0}$. We will use the same notations. We choose a natural approximating sequence $P^{(n)}, I^{(n)}$. Namely, we order all points by their masses and $P^{(n)}$ is concentrated on the set of all points whose masses are not less than $\frac{1}{n}$. $I^{(n)}$ is defined in an analogous way. We use the following lemma which will be proved later.

Lemma $4^{\prime}$. For any $\beta>0$ take $S_{\beta}=\left\{x: P_{0}(\{x\}) \geqq \beta\right\}$. Then $\varphi_{t}^{(n)}(y)$ converges uniformly to $\varphi_{t}(y)$ on bounded subsets of $S_{\beta} \times R^{+}$as $n \rightarrow \infty$.

The proof of (D1) basically remains the same as in the continuous case. So the main problem is to prove (D2).

Fix $\beta, \varepsilon$. Assume that $(y, \tau), y \in S_{\beta}$ is such that for all large enough $n$,

$$
\left|u_{0}\left(C_{\tau}(y)\right)-u_{0}^{(n)}\left(C_{\tau}^{(n)}(y)\right)\right|>\varepsilon .
$$

We have

$$
\begin{aligned}
\left|u_{0}^{(n)}\left(C_{\tau}^{(n)}(y)\right)-u_{0}\left(C_{\tau}^{(n)}(y)\right)\right| & =\left|\frac{\int_{C_{\tau}^{(n)}(y)} u(\eta ; 0) d P_{0}^{(n)}(\eta)}{\int_{C_{\tau}^{(n)}(y)} d P_{0}^{(n)}(\eta)}-\frac{\int_{C_{\tau}^{(n)}(y)} u(\eta ; 0) d P_{0}(\eta)}{\int_{C_{\tau}^{(n)}(y)} d P_{0}(\eta)}\right| \\
& \leqq \frac{\text { const } \cdot \delta_{n}}{\beta^{2}},
\end{aligned}
$$

where $\delta_{n} \rightarrow 0$ as $n \rightarrow \infty$. Choose $n$ so large that $\frac{\text { const } \cdot \delta_{n}}{\beta^{2}}<\frac{\varepsilon}{2}$. Then

$$
\left|u_{0}\left(C_{\tau}(y)\right)-u_{0}\left(C_{\tau}^{(n)}(y)\right)\right|>\frac{\varepsilon}{2} .
$$

Without any loss of generality we may assume that $C_{\tau}(y)=\Delta_{1} \cup \Delta_{2}, C_{\tau}^{(n)}(y)=$ $\Delta_{2} \cup \Delta_{3}$ and $\Delta_{1}\left(\Delta_{3}\right)$ lies to the left (right) of $\Delta_{2}$. We can write

$$
\begin{aligned}
u_{0}\left(C_{\tau}(y)\right) & =\alpha u_{0}\left(\Delta_{1}\right)+(1-\alpha) u_{0}\left(\Delta_{2}\right), \\
u_{0}\left(C_{\tau}^{(n)}(y)\right) & =\xi u_{0}\left(\Delta_{3}\right)+(1-\xi) u_{0}\left(\Delta_{2}\right) .
\end{aligned}
$$

We claim that there is a constant $K$, such that either $\alpha>K \varepsilon$ or $\xi>K \varepsilon$. Indeed if it were not true, we would have

$$
\left|u_{0}\left(C_{\tau}(y)\right)-u_{0}\left(C_{\tau}^{(n)}(y)\right)\right| \leqq 4 K\left(\max \left|u_{0}\right|\right) \cdot \varepsilon<\frac{\varepsilon}{2},
$$

provided that $K=\frac{1}{8 \max \left|u_{0}\right|}$. The last inequality contradicts (4.25).

Consider for definiteness the case $\alpha>K \varepsilon$, i.e. $P_{0}\left(\Delta_{1}\right)>K \varepsilon P_{0}\left(\Delta_{1} \cup \Delta_{2}\right) \geqq K \beta \varepsilon$.

We will need an estimate for the time needed to absorb an essential part of $\Delta_{1}$ by the atom of $\varphi_{\tau}^{(n)}$ in the dynamics with data $\left(P_{0}^{n}, I_{0}^{n}\right)$.

Let $\Delta_{1}=J_{1} \cup J_{2}$, where $J_{2}$ is the part which has been absorbed at $t=\tau+\gamma$, and $P_{0}\left(J_{1}\right)>\frac{K \beta \varepsilon}{2}$. Then

$$
\begin{aligned}
& C_{J_{1}}(t)>\varphi_{\tau}(y)+\gamma u_{0}\left(J_{1}\right) \geqq \varphi_{\tau}(y)+\gamma u_{0}\left(\Delta_{1}\right), \\
& C_{J_{1}}^{(n)}(t) \geqq \varphi_{\tau}(y)+\gamma u_{0}\left(\Delta_{1}\right)+o(1) .
\end{aligned}
$$


Here $o(1)$ means quantities which tend to zero as $n \rightarrow \infty$ and $\beta, \varepsilon$ remain fixed. We also have

$$
C_{\Delta_{2} \cup \Delta_{3}}^{(n)}(t)=\varphi_{\tau}^{(n)}(y)+\gamma u_{0}\left(C_{\tau}^{(n)}(y)\right) \leqq \varphi_{\tau}^{(n)}(y)+\gamma u_{0}\left(C_{\tau}^{(n)}(y)\right)+o(1) .
$$

By assumption at time $t$, the set $J_{1}$ is not absorbed by the atom which at time $\tau$ were at $\varphi_{\tau}^{(n)}$, but $J_{2}$ is, we can write

$$
\varphi_{\tau}(y)+\gamma u_{0}\left(\Delta_{1}\right) \leqq \varphi_{\tau}^{(n)}(y)+\gamma u_{0}\left(C_{\tau}^{(n)}(y)\right)+o(1)
$$

or

$$
\gamma\left(u_{0}\left(\Delta_{1}\right)-u_{0}\left(C_{\tau}^{(n)}(y)\right)\right) \leqq o(1)
$$

Since

$$
u_{0}\left(\Delta_{1}\right)-u_{0}\left(C_{\tau}^{(n)}(y)\right) \geqq u_{0}\left(C_{\tau}(y)\right)-u_{0}\left(C_{\tau}^{(n)}(y)\right)>\frac{\varepsilon}{2},
$$

we conclude

$$
\gamma \leqq o(1) .
$$

Define $S^{\beta}=\left\{\tau \in\left[t_{1}, t_{2}\right]\right.$ one can find $y \in L \cap S_{\beta}$ so that (4.24) holds $\}$. As in the continuous case, we have

$$
\left|S^{\beta}\right| \leqq o(1) \text {. }
$$

Now take $g \in C_{0}^{1}\left(R^{1}\right)$. We can write

$$
\begin{aligned}
& \left|\int_{t_{1}}^{t_{2}} d t \int g^{\prime}(\eta)\left(u_{\tau}^{(n)}(\eta)\right)^{2} d P_{0}^{(n)}(\eta)-\int_{t_{1}}^{t_{2}} d t \int g^{\prime}(\eta)\left(u_{\tau}(\eta)\right)^{2} d P_{0}(\eta)\right| \\
& \leqq o(1)+\left|\int_{t_{1}}^{t_{2}} d t \int g^{\prime}\left(\varphi_{\tau}(\eta)\right)\left[\left(\omega^{(n)}(\eta ; \tau)\right)^{2}-(\omega(\eta ; \tau))^{2}\right] d P_{0}(\eta)\right| \\
& \leqq o(1)+\text { const }\left|\int_{t_{1}}^{t_{2}} d t \int_{P_{0}(\eta)<\beta} d P_{0}(\eta)\right| \\
& +\left|\int_{S^{\beta}} d t \int_{S_{\beta}} g^{\prime}\left(\varphi_{\tau}(\eta)\right)\left(\left(\omega^{(n)}(\eta ; \tau)\right)^{2}-(\omega(\eta ; \tau))^{2}\right) d P_{0}(\eta)\right| \\
& \left.+\mid \int_{\left[t_{1} ; t_{2}\right] \backslash S^{\beta}} \int_{S^{\beta}} g^{\prime}\left(\varphi_{\tau}(\eta)\right)\left(\omega^{(n)}(\eta ; \tau)\right)^{2}-(\omega(\eta ; \tau))^{2}\right) d P_{0}(\eta) \mid \\
& \leqq o(1)+C(\beta)+\text { const }\left|S^{\beta}\right|+\text { const } \cdot \varepsilon=o(1)+C(\beta)+\text { const } \cdot \varepsilon .
\end{aligned}
$$

The rest of the proof is the same as in the continuous case.

Proof of Lemma 4'. We will follow the proof of Lemma 4. Assume that one can find a sequence $\left\{y^{(n)}\right\}$ such that $P_{0}\left(y^{(n)}\right) \geqq \beta$ and

$$
\left|\varphi_{t}^{(n)}\left(y^{(n)}\right)-\varphi_{t}\left(y^{(n)}\right)\right|>\varepsilon
$$


for some $\varepsilon>0$ and an infinite sequence of values of $n$. For each of these $n$ one can find three adjoining intervals $\Delta_{1}^{(n)}, \Delta_{2}^{(n)}, \Delta_{2}^{(n)}$ such that $C_{\xi(n)}\left(y^{(n)}\right)=$ $\Delta_{1}^{(n)} \cup \Delta_{2}^{(n)}, C_{\xi}\left(y^{(n)}\right)=\Delta_{2}^{(n)} \cup \Delta_{3}^{(n)}$. Let us assume that we have

$$
\varphi_{t}^{(n)}\left(y^{(n)}\right)<\varphi_{t}\left(y^{(n)}\right) .
$$

Arguing as above we conclude that either $P_{0}\left(\Delta_{1}^{(n)}\right)>K_{1} \beta \varepsilon$, or $P_{0}\left(\Delta_{3}^{(n)}\right)>K_{1} \beta \varepsilon$ for some constant $K_{1}$. For definiteness let us assume $P_{0}\left(\Delta_{1}^{(n)}\right)>K_{1} \beta \varepsilon$. Then GVP gives

$$
\begin{aligned}
& C_{\Delta_{1}^{(n)}}(t)<C_{\Delta_{2}^{(n)} \cup \Delta_{3}^{(n)}}(t)=\varphi_{t}\left(y^{(n)}\right) \leqq C_{\Delta_{2}^{(n)}}(t), \\
& C_{\Delta_{1}^{(n)}}^{(n)}(t) \geqq C_{\Delta_{1}^{(n)} \cup \Delta_{2}^{(n)}}^{(n)}(t)=\varphi_{t}^{(n)}\left(y^{(n)}\right) \geqq C_{\Delta_{2}^{(n)}}^{(n)}(t),
\end{aligned}
$$

or

$$
\begin{aligned}
& C_{\Delta_{1}^{(n)}}(t)<\varphi_{t}\left(y^{(n)}\right) \leqq C_{\Delta_{2}^{(n)}}(t), \\
& C_{\Delta_{1}^{(n)}}^{(n)}(t) \geqq \varphi_{t}^{(n)}\left(y^{(n)}\right) \geqq C_{\Delta_{2}^{(n)}}^{(n)}(t) .
\end{aligned}
$$

Since $P_{0}\left(\Delta_{1}^{(n)}\right)>K \beta \varepsilon, P_{0}\left(\Delta_{2}^{(n)}\right)>\beta$, we have

$$
\begin{aligned}
& \left|C_{\Delta_{1}^{(n)}}^{(n)}(t)-C_{\Delta_{1}^{(n)}}(t)\right| \leqq o(1), \\
& \left|C_{\Delta_{2}^{(n)}}^{(n)}(t)-C_{\Delta_{2}^{(n)}}(t)\right| \leqq o(1) .
\end{aligned}
$$

This contradicts (4.26).

\section{The Gravitationally Interacting Case}

In this section we prove the GVP for (1.2) and Theorem 2. Since most of the argument is parallel to the ones in Sects. $2-4$, we will only summarize the key steps.

Lemma 5. The family of partitions $\left\{\xi_{t}\right\}_{t>0}$ is decreasing, i.e. for $0<t^{\prime}<t$, $\partial \xi_{t} \subseteq \partial \xi_{t^{\prime}}$

Proof. Assume to the contrary that there exists $y \in \partial \xi_{t}$, but $y \bar{\epsilon} \partial \xi_{t^{\prime}}$. Then for some $y^{-}, y^{+}, y^{-}<y<y^{+}$, we should have

$$
\begin{aligned}
& \frac{\int_{\left[y^{-}, y\right)}\left(\eta+t^{\prime} u_{0}(\eta)\right) d P_{0}(\eta)}{\int_{\left[y^{-}, y\right)} d P_{0}(\eta)}+\frac{\left(t^{\prime}\right)^{2}}{2}\left(P_{0}(y, \infty)-P_{0}\left(-\infty, y^{-}\right)\right) \\
& \geqq \frac{\int_{\left[y, y^{+}\right]}\left(\eta+t^{\prime} u_{0}(\eta)\right) d P_{0}(\eta)}{\int_{\left[y, y^{+}\right]} d P_{0}(\eta)}+\frac{\left(t^{\prime}\right)^{2}}{2}\left(P_{0}\left(y^{+}, \infty\right)-P_{0}(-\infty, y)\right) .
\end{aligned}
$$


Consider two quadratic functions $q_{1}(s), q_{2}(s)$ defined by

$$
\begin{aligned}
q_{1}(s) & =\frac{\int_{\left[y^{-}, y\right)}\left(\eta+s u_{0}(\eta)\right) d P_{0}(\eta)}{\int_{\left[y^{-}, y\right)} d P_{0}(\eta)}+\frac{s^{2}}{2}\left(P_{0}[y, \infty)-P_{0}\left(-\infty, y^{-}\right)\right) \\
& =A^{-}+s B^{-}+\frac{s^{2}}{2} C^{-} \\
q_{2}(s) & =\frac{\int_{\left[y, y^{+}\right]}\left(\eta+s u_{0}(\eta)\right) d P_{0}(\eta)}{\int_{\left[y, y^{+}\right]} d P_{0}(\eta)}+\frac{s^{2}}{2}\left(P_{0}\left[y^{+}, \infty\right)-P_{0}(-\infty, y)\right) \\
& =A^{+}+s B^{+}+\frac{s^{2}}{2} C^{+}
\end{aligned}
$$

For sufficiently small $s$, we have $A^{-}<A^{+}$and $q_{1}(s)<q_{2}(s)$, while $q_{1}\left(t^{\prime}\right) \geqq q_{2}\left(t^{\prime}\right)$. Since $C^{-}-C^{+}=P_{0}\left[y^{-}, y^{+}\right]>0, A^{-}-A^{+}<0$, one of the two roots of the equation $q_{1}(s)=q_{2}(s)$ is negative. Since there is one positive root in $\left(0, t^{\prime}\right)$, no roots exist in $\left(t^{\prime}, \infty\right)$. Therefore we have $q_{1}(s)>q_{2}(s)$ for $s>t^{\prime}$, contradicting the assumption that $q_{1}(t)<q_{2}(t)$.

There is also an obvious analog of Lemma 2 for (1.2). We omit the details of that.

Next we study the discrete case, using the notations of Sect. 1. In the present situation, the center of masses of the particles $\left\{x_{i}^{0}, x_{i+1}^{0}, \ldots, x_{j}^{0}\right\}$ becomes

$$
C_{i, j}(t)=\frac{\sum_{i \leqq l \leqq j} m_{l}^{0}\left(x_{\ell}^{0}+t v_{l}^{0}\right)}{\sum_{i \leqq l \leqq j} m_{l}^{0}}+\frac{t^{2}}{2}\left(\sum_{l>j} m_{l}^{0}-\sum_{l<i} m_{l}^{0}\right) .
$$

Lemma 6. Let $G \in \xi_{t}, G=\left\{x_{i}^{0}, j^{\prime} \leqq i \leqq j^{\prime \prime}\right\}$. Then for any $i, j^{\prime} \leqq i \leqq j^{\prime \prime}-1$, we have

$$
C_{j^{\prime}, i}(t) \geqq C_{i+1, j^{\prime \prime}}(t)
$$

Proof. We proceed as in the proof of Lemma 3. The key step is to show that if two groups of particles $\left\{\left(x_{i}^{0}, v_{i}^{0}, m_{i}^{0}\right), j_{1} \leqq i \leqq j\right\}$ and $\left\{\left(x_{i}^{0}, v_{i}^{0}, m_{i}^{0}\right), j+1 \leqq i \leqq j_{2}\right\}$ merge at time $t^{\prime}$, then

$$
C_{j_{1}, j_{2}}(s) \leqq C_{j_{1}, j}(s), \quad s \geqq t^{\prime}
$$

Consider again two quadratic functions $q_{1}(s)=C_{j_{1}, j}(s)=A_{1}+B_{1} s+C_{1} \frac{s^{2}}{2}, q_{2}(s)=$ $C_{j+1, j_{2}}(s)=A_{2}+B_{2} s+C_{2} \frac{s^{2}}{2}$. Obviously we have $A_{1}-A_{2}<0, C_{1}-C_{2}=\sum_{i>j} m_{i}^{0}$ $-\sum_{i>j_{1}} m_{i}^{0}-\sum_{i>j_{2}} m_{i}^{0}+\sum_{i<j+1} m_{i}^{0}=\sum_{j_{1} \leqq i \leqq j_{2}} m_{i}^{0}>0$. Therefore the quadratic equation $q_{1}(s)-q_{2}(s)=0$ has one negative root. Since there is already one root in $\left(0, t^{\prime}\right)$, there cannot be any more roots in $\left(t^{\prime}, \infty\right)$. Therefore, we have

$$
C_{j_{1}, j}(s) \geqq C_{j+1, j_{2}}(s), \quad \text { for } s>t^{\prime}
$$

This implies (5.4). 
Similarly we can formulate

Theorem $2^{\prime} . x_{j}^{0}$ is the left end point of an element of $\xi_{t}$ iff

$$
\max _{j^{\prime}<j} C_{j^{\prime}, j-1}(t)<\min _{j \leqq j^{\prime \prime}} C_{j, j^{\prime \prime}}(t) .
$$

We now prove that the adhesion dynamics of gravitationally interacting particles gives rise to weak solutions of (1.2). Since the proof of $\left(\mathrm{D} 1^{\prime}\right)$ is entirely the same as the one for (D1), we will concentrate on (D2'). Again we only have to prove $\left(\mathrm{D} 2^{\prime}\right)$ for the case when there is no collision in $\left(t_{1}, t_{2}\right)$, and at $t=t_{2}$, a group of particles, say indexed by $i_{1}, \ldots, i_{2}$, collide to form the new particle at $x\left(t_{2}\right)$. Let $h_{i}=\sum_{j>i} m_{j}-\sum_{j<i} m_{j}$, then we have

$$
\begin{gathered}
\frac{d}{d \tau} v_{i}(\tau)=h_{i} \\
\int_{t_{1}}^{t_{2}} d \tau \int g^{\prime}(\eta) u(\eta, \tau) d I_{\tau}(\eta)+\int_{t_{1}}^{t_{2}} d \tau \int g(\eta)\left(P_{\tau}(\eta, \infty)-P_{\tau}(-\infty, \tau)\right) d P_{\tau}(\eta) \\
=\int_{t_{1}}^{t_{2}} d \tau \sum_{i=1}^{N} g^{\prime}\left(x_{i}(\tau)\right) v_{i}^{2}(\tau) m_{i}+\int_{t_{1}}^{t_{2}} d \tau \sum_{i=1}^{N} g\left(x_{i}(\tau)\right) h_{i} m_{i} \\
=\int_{t_{1}}^{t_{2}} d \tau \sum_{i=1}^{N} m_{i} v_{i}(\tau) \frac{d}{d \tau} g\left(x_{i}(\tau)\right)+\int_{t_{1}}^{t_{2}} d \tau \sum_{i=1}^{N} g\left(x_{i}(\tau)\right) h_{i} m_{i} \\
=\sum_{i=1}^{N} g\left(x_{i}\left(t_{2}\right)\right) m_{i} v_{i}\left(t_{2}\right)-\sum_{i=1}^{N} g\left(x_{i}\left(t_{1}\right)\right) m_{i} v_{i}\left(t_{1}\right) \\
-\int_{t_{1}}^{t_{2}} d \tau \sum_{i=1}^{N} m_{i} h_{i} g\left(x_{i}(\tau)\right)+\int_{t_{1}}^{t_{2}} d \tau \sum_{i=1}^{N} g\left(x_{i}(\tau)\right) h_{i} m_{i} \\
=\int g(\eta) d I_{t_{2}}(\eta)-\int g(\eta) d I_{t_{1}}(\eta)
\end{gathered}
$$

To pass to the continuum limit, we follow the same steps as in the proof of Theorem 1. Some care has to be taken at the boundary of $\operatorname{supp}\left\{P_{0}\right\}$ since $\rho_{0}^{\prime}>$ const $>0$ no longer holds. This can be done by making the set $\left\{x=\rho_{0}^{\prime}(x)<\right.$ const \} small by choosing the constant small.

\section{The Modified Gravitational Model}

In this section we study a variant of (1.2) for which some other weighted velocity, instead of momentum, is conserved.

$$
\left\{\begin{aligned}
\rho_{t}+(\rho u)_{x} & =0 \\
u_{t}+\left(\frac{u^{2}}{2}\right)_{x} & =-g_{x} \\
g_{x x} & =\rho .
\end{aligned}\right.
$$

This model, or more precisely its multidimensional version, is an approximation of the basic equations in astrophysics for the dynamic evolution of matter in which effects of Hubble expansion are neglected [P,GS]. For this system, we will prove 
not only existence, but also uniqueness of solutions for the initial value problem. We will also obtain precise information on the characteristics of this system. This information will be used in the second part of this paper to study the behavior of solutions with random initial data.

As in Sect. 5, we assume that $\rho(x, 0)=\rho_{0}(x) \geqq 0, u(x, 0)=u_{0}(x)$. Moreover, we will assume $\rho_{0} \in L^{1}$, and (A3) holds. Without loss of generality, we let $\int \rho_{0} d x=1$.

Let $h(x, t)=\int_{-\infty}^{x} \rho(\eta, t) d \eta-\frac{1}{2}$. Obviously, for any reasonable solution of (6.1), we should have $h( \pm \infty, t)= \pm 1 / 2$.

In the following we will deal with solutions having the property that for any $t>0, u(\cdot, t)$ is of bounded variation on any compact interval; $\rho(\cdot, t)$ is a nonnegative Radon measure. We interpret the product $u \rho$ in the sense of Volpert [V]:

$$
u \rho=\bar{u} \rho=\bar{u} h_{x},
$$

where $\bar{u}$ is the symmetric mean of $u: \bar{u}(x, t)=u(x, t)$ if $(x, t)$ is a point of approximate continuity of $u$. At jump points, $\bar{u}(x, t)=\frac{1}{2}\left(l_{+} u(x, t)+l_{-} u(x, t)\right)$, where $l_{+} u(x, t)$ and $l_{-} u(x, t)$ are the limits of $u$ at $(x, t)$ from the two sides of the jump. Given this, we can rewrite (6.1) in an equivalent form,

$$
\left\{\begin{array}{c}
h_{t}+\bar{u} h_{x}=0 \\
u_{t}+\left(\frac{u^{2}}{2}\right)_{x}=-h,
\end{array}\right.
$$

where the first equation in (6.3) is understood in the sense of measures. We let $h_{0}(y)=h(y, 0)$. We assume $\rho_{0}, u_{0} \in C^{0}$.

A special property of (6.3) is that for smooth solutions, the characteristics can be written down very easily:

$$
\left\{\begin{aligned}
x & =y+t u_{0}(y)-\frac{t^{2}}{2} h_{0}(y) \\
h(x, t) & =h_{0}(y) \\
u(x, t) & =u_{0}(y)-t h_{0}(y)
\end{aligned}\right.
$$

From (6.4), we get

$$
x-u(x, t) t=y+\frac{t^{2}}{2} h_{0}(y) .
$$

Since the right-hand side of (6.5) is a strictly increasing function of $y$, we can invert it to get

$$
y=G(x-u(x, t) t, t)
$$

where $G(z, t)$ solves

$$
z=G+\frac{t^{2}}{2} h_{0}(G)
$$

Therefore, we have

$$
h(x, t)=h_{0}(G(x-u(x, t) t, t)) .
$$

This suggests the following:

Lemma 7. Let $(h, u)$ be a solution of (6.3) such that $u$ is locally $B V$ on $R^{1} \times$ $(0, \infty)$. Then $u$ is also a solution of

$$
u_{t}+\left(\frac{u^{2}}{2}\right)_{x}=-h_{0}(G(x-u t, t)) .
$$


Conversely, if $u$ is a solution of (6.9) with locally bounded variations on $R^{1} \times$ $(0, \infty)$, and let $h(x, t)=h_{0}(G(x-u t, t))$, then $(h, u)$ is also a solution of $(6.3)$.

Proof. In the proof we will use $\hat{f}(u)$ to denote the functional composition of $f$ and $u: \hat{f}(u)=\int_{0}^{1} f\left(\alpha l_{-} u+(1-\alpha) l_{+} u\right) d \alpha$.

Let $(h, u)$ be a solution of (6.3) such that $u$ is locally BV on $R^{1} \times(0, \infty)$. Using BV calculus [V], we see that $u$ and $h$ also satisfy

$$
u_{t}+\bar{u} u_{x}=-h, h_{t}+\bar{u} h_{x}=0
$$

in the sense of measures.

Let $g(x, t)=h_{0}(G(x-u t, t))$. Again using BV calculus, we get

$$
\begin{aligned}
g_{t}+\bar{u} g_{x} & =\widehat{h_{0}^{\prime}}\left\{G_{t}+\widehat{G}_{z}\left(-u-u_{t} t\right)+\bar{u} \widehat{G}_{z}\left(1-u_{x} t\right)\right\} \\
& =\widehat{h_{0}^{\prime}}\left\{G_{t}-t \widehat{G}_{z}\left(u_{t}+\bar{u} u_{x}\right)\right\}=\widehat{h}_{0}^{\prime}\left\{G_{t}+t \widehat{G}_{z} h\right\}
\end{aligned}
$$

The final expression only involves locally bounded functions. Therefore it can be simply written as $h_{0}^{\prime}\left\{G_{t}+t G_{z} h\right\}$. Since

$$
G_{t}(z, t)=-\frac{t h_{0}(G)}{1+\frac{t^{2}}{2} h_{0}^{\prime}(G)}, \quad G_{z}(z, t)=\frac{1}{1+\frac{t^{2}}{2} h_{0}^{\prime}(G)},
$$

we obtain

$$
g_{t}+\bar{u} g_{x}=-\frac{h_{0}^{\prime}(G) t}{1+\frac{t^{2}}{2} h_{0}^{\prime}(G)}\{g-h\} .
$$

Let $e=g-h$. We have

$$
e_{t}+\bar{u} e_{x}=-\frac{h_{0}^{\prime}(G) t}{1+\frac{t^{2}}{2} h_{0}^{\prime}(G)} e
$$

and $e(x, 0)=0$. (6.9).

This implies that $e \equiv 0$. Hence $h(x, t)=h_{0}(G(x-u t, t))$, i.e. $u$ is a solution of

Conversely assume $u$ is solution of (6.9) with locally bounded variation. We let $h(x, t)=h_{0}(G(x-u t, t))$. Then a similar calculation shows that $h$ satisfies

$$
h_{t}+\bar{u} h_{x}=0 \text {. }
$$

Hence $(h, u)$ solves $(6.3)$.

Now the problem is reduced to (6.9) which is a scalar equation. We are ready to prove:

Theorem 3. Assume that $\rho_{0}$ and $u_{0}$ are continuous and satisfy (A3). Then there exists a weak solution $(h, u)$ of $(6.3)$ with initial data $\left(h_{0}, u_{0}\right)$ such that

$$
u_{x}<\frac{1}{t}
$$

in the distributional sense, and $u$ is bounded locally and has locally bounded variations in $R^{1} \times(0, \infty)$. Furthermore, such solutions are unique. 
Proof. This theorem follows more or less from standard arguments on conservation laws [V], with some care on cutting off the growth at infinity. Here we will not repeat this argument. We will only indicate how (6.12) can be derived from the viscosity approximation.

Consider

$$
u_{t}^{\varepsilon}+\left(\frac{\left(u^{\varepsilon}\right)^{2}}{2}\right)_{x}=-h_{0}\left(G\left(x-u^{\varepsilon} t, t\right)\right)+\varepsilon u_{x x}^{\varepsilon} .
$$

Let $\xi=u_{x}^{\varepsilon}$. Differentiating both sides of (6.13), we get

$$
\xi_{t}+u \xi_{x}+\xi^{2}=-h_{0}^{\prime} G_{z}(1-\xi t)+\varepsilon \xi_{x x} .
$$

The standard maximum principle implies that, if we let $f(t)=\sup _{x} \xi(x, t)$, then

$$
\frac{d}{d t} f \leqq h_{0}^{\prime} G_{z}(f t-1)-f^{2}
$$

Since $h_{0}^{\prime} G_{z} \geqq 0$, we obtain

$$
f<\frac{1}{t}
$$

Taking the limit as $\varepsilon \rightarrow 0$, we get (6.12).

Next we summarize the main results on the generalized characteristics of (6.9), drawing mainly from the work of Dafermos [D]. A Lipschitz curve $\xi(\cdot):[a, b] \rightarrow$ $R^{1}$ is called a generalized characteristic if $\xi(t) \in[u(\xi(t)+, t), u(\xi(t)-, t)]$, for a.e. $t \in[a, b]$. It is called a genuine characteristic if $u(\xi(t)-, t)=u(\xi(t)+, t)$, for a.e. $t \in[a, b]$. The following holds if $u$ is locally $\mathrm{BV}$ on $R^{1} \times(0, \infty)$ :

(1) If $\xi$ is a generalized characteristic on $[a, b]$, then for a.e. $t \in[a, b]$

$$
\dot{\xi}(t)=\left\{\begin{array}{l}
u(\xi(t) \pm, t), \quad \text { if } u(\xi(t)+, t)=u(\xi(t)-, t) \\
\frac{1}{2}(u(\xi(t)+, t)+u(\xi(t)-, t))=\bar{u}(\xi(t)), \quad \text { if not }
\end{array} .\right.
$$

(2) Given $(x, t) \in R^{1} \times(0, \infty)$, there exists a funnel of backward generalized characteristics from $(x, t)$ to the initial time $t=0$. Among them, there exists a unique minimal and a unique maximal one. They are both genuine.

(3) Let $\xi$ be a genuine characteristic. Then by modifying on a measure zero set, one can extend $u(\xi(t)-, t) \stackrel{\text { a.e. }}{=} u(\xi(t)+, t)$ to a $C^{1}$ function $\bar{u}(t)$, such that

$$
\frac{d \xi(t)}{d t}=\bar{u}(t), \quad \frac{d \bar{u}(t)}{d t}=h_{0}(G(\xi(t)-\bar{u}(t) t, t)) .
$$

In particular, if $(\bar{x}, \bar{t})$ is point of continuity, i.e. $u(\bar{x}+, \bar{t})=u(\bar{x}-, \bar{t})$, then from $(\bar{x}, \bar{t})$, there is a unique backward generalized characteristic. Moreover, (6.15) holds.

Recall that $h(x, t)=h_{0}(G(x-u(x, t) t, t))$. Along a genuine characteristic, we have

$$
\begin{aligned}
\frac{d}{d t} h(\xi(t), t) & =h_{0}^{\prime}\left\{G_{t}+G_{z}\left(\frac{d \xi}{d t}-\bar{u}-t \frac{d \bar{u}}{d t}\right)\right\} \\
& =h_{0}^{\prime}\left\{G_{t}+t h_{0}(G) G_{z}\right\} \\
& =0 .
\end{aligned}
$$




\section{Part II. Behavior with Random Initial Data}

\section{Behavior of Solutions with Random Initial Data for Systems (1.1) and (1.2)}

This part is devoted to the study of the qualitative behavior of solutions constructed above when the initial data are random. We prove that the systems (1.1), (1.2), and (1.3) share the following common feature: For continuous but nowhere differentiable random initial data, almost surely, the solution $u(x, t)$ becomes discontinuous for any $t>0$, the set of discontinuities (shocks) is dense (on the appropriate intervals), and almost all masses are absorbed by the shocks. In particular for any $t>0, \rho(\cdot, t)$ becomes a discrete measure even though it may have a smooth distribution at $t=0$. Such behavior was, to some extent, anticipated by Zeldovich [Z], as part of his proposal for the formation of large scale structures in the universe. Technically, we extend some of the arguments in [S].

Let $Q$ be the probability distribution of initial velocities, $u(x ; 0)=u_{0}(x)$. We assume

(Q1) $Q$ is defined on the Borel $\sigma$-algebra of the space of continuous functions on $R^{1}$, and for a.e. $u_{0}$ (with respect to $Q$ ),

$$
\lim _{|x| \rightarrow \infty} \frac{u_{0}(x)}{|x|}=0
$$

(Q2) For a.e. $u_{0}$, the following holds: for any $\eta_{0} \in R^{1}$,

$$
\begin{aligned}
& \lim _{h \rightarrow 0+} \sup \frac{\int_{\eta_{0}-h}^{\eta_{0}}\left(u(\eta ; 0)-u\left(\eta_{0} ; 0\right)\right) d \eta}{h^{2}}=\infty, \\
& \lim _{h \rightarrow 0+} \inf \frac{\int_{\eta_{0}}^{\eta_{0}+h}\left(u(\eta ; 0)-u\left(\eta_{0} ; 0\right)\right) d \eta}{h^{2}}=-\infty .
\end{aligned}
$$

Concerning $\rho_{0}$, the initial distribution of masses, we will assume either

or

(1) $\rho_{0} \in C^{1}\left(R^{1}\right), \quad 0<$ const $\leqq \rho_{0} \leqq$ const $<\infty, \quad\left|\rho^{\prime}\right| \leqq$ const $<\infty$

(2) $\rho_{0} \in C^{1}\left(R^{1}\right), \quad \rho_{0}(x)>0$ for $x \in(a, b)$, and $\rho_{0}(x)=0$, for $x \bar{\epsilon}(a, b)$, $\left|\rho_{0}^{\prime}(x)\right| \leqq$ const $<\infty$. For (1.2) we will only consider the case (2).

Theorem 4. For a.e. $u_{0}$, the weak solutions of (1.1) and (1.2) constructed by GVP have the following properties: For any $t>0$,

(a) The measure $P_{t}$ is a pure point measure, i.e. $P_{t}(x)=\sum_{i} m_{i} \delta\left(x-x_{i}\right)$, $\left(m_{i}>0\right)$.

(b) The closure of the set $\left\{x_{i}\right\}$ is either $R^{1}$ in the case of (1), or a closed interval $I(t)$ in the case of $(2)$.

Proof. We will only prove this theorem for (1.1). The modifications required for (1.2) are obvious. 
We will show that for a.e. $u_{0}$, the partition $\xi_{t}$ given by GVP has the following properties:

i) There exists a countable set of elements of $\xi_{t}$ which are intervals of positive length.

ii) The union of these intervals is a set of full measure

iii) Between any two of these intervals there are infinitely many similar intervals.

Clearly (i)-(iii) implies the theorem.

Fix $y_{0} \neq a, b$ and take $u(\cdot ; 0)$ satisfying (Q1). Assume that $y_{0}$ is the left end point of an element of $\xi_{t}$, i.e. for any $h_{1}, h_{2}>0$,

$$
\frac{\int_{y_{0}-h_{1}}^{y_{0}}(\eta+t u(\eta ; 0)) \rho_{0}(\eta) d \eta}{\int_{y_{0}-h_{1}}^{y_{0}} \rho_{0}(\eta) d \eta} \leqq \frac{\int_{y_{0}}^{y_{0}+h_{2}}(\eta+t u(\eta ; 0)) \rho_{0}(\eta) d \eta}{\int_{y_{0}}^{y_{0}+h_{2}} \rho_{0}(\eta) d \eta}
$$

We will show that the Q-probability of such $u(\cdot ; 0)$ is zero. Indeed we can rewrite (7.1) as

$$
\begin{gathered}
\frac{\int_{y_{0}-h_{1}}^{y_{0}}\left(\eta-y_{0}\right) \rho_{0}(\eta) d \eta}{\int_{y_{0}-h_{1}}^{y_{0}} \rho_{0}(\eta) d \eta}+t \frac{\int_{y_{0}-h_{1}}^{y_{0}}\left(u(\eta ; 0)-u\left(y_{0} ; 0\right)\right) \rho_{0}(\eta) d \eta}{\int_{y_{0}-h_{1}}^{y_{0}} \rho_{0}(\eta) d \eta} \\
\leqq \frac{\int_{y_{0}}^{y_{0}+h_{2}}\left(\eta-y_{0}\right) \rho_{0}(\eta) d \eta}{\int_{y_{0}}^{y_{0}+h_{2}} \rho_{0}(\eta) d \eta}+t \frac{\int_{y_{0}}^{y_{0}+h_{2}}\left(u(\eta ; 0)-u\left(y_{0} ; 0\right)\right) \rho_{0}(\eta) d \eta}{\int_{y_{0}}^{y_{0}+h_{2}} \rho_{0}(\eta) d \eta}
\end{gathered}
$$

Since $\rho_{0}\left(y_{0}\right)>0$, we have

$$
\begin{gathered}
\frac{\int_{y_{0}-h_{1}}^{y_{0}}\left(u(\eta ; 0)-u\left(y_{0} ; 0\right)\right) \rho_{0}(\eta) d \eta}{\int_{y_{0}-h_{1}}^{y_{0}} \rho_{0}(\eta) d \eta}=\frac{\rho_{0}\left(y_{0}\right) \int_{y_{0}-h_{1}}^{y_{0}}\left(u(\eta ; 0)-u\left(y_{0} ; 0\right)\right) d \eta}{\rho_{0}\left(y_{0}\right) h_{1}+\int_{y_{0}-h_{1}}^{y_{0}}\left(\rho_{0}(\eta)-\rho_{0}\left(y_{0}\right)\right) d \eta} \\
\quad+\frac{\int_{y_{0}-h_{1}}^{y_{0}}\left(u(\eta ; 0)-u\left(y_{0} ; 0\right)\right)\left(\rho_{0}(\eta)-\rho_{0}\left(y_{0}\right)\right) d \eta}{\rho_{0}\left(y_{0}\right) h_{1}+\int_{y_{0}-h_{1}}^{y_{0}}\left(\rho_{0}(\eta)-\rho_{0}\left(y_{0}\right)\right) d \eta} \\
=\frac{\int_{y_{0}-h_{1}}^{y_{0}}\left(u(\eta ; 0)-u\left(y_{0} ; 0\right)\right) d \eta}{h_{1}}(1+o(1))+O\left(h_{1}\right) .
\end{gathered}
$$

Similar estimates hold for the integrals on $\left(y_{0}, y_{0}+h_{2}\right)$. Therefore, we can write (7.1) as

$$
\begin{aligned}
& \frac{\int_{y_{0}-h_{1}}^{y_{0}}\left(u(\eta ; 0)-u\left(y_{0} ; 0\right)\right) d \eta}{h_{1}}(1+o(1))+O\left(h_{1}\right) \\
& \leqq \frac{\int_{y_{0}}^{y_{0}+h_{2}}\left(u(\eta ; 0)-u\left(y_{0} ; 0\right)\right) d \eta}{h_{2}}(1+o(1))+O\left(h_{2}\right) .
\end{aligned}
$$

From (Q2), this can only hold on a $Q$-measure 0 set of $u(\cdot ; 0)$.

Next we consider the measurable space of $\left(u(\cdot ; 0), y_{0}\right)$ equipped with the product measure of $Q$ and the Lebesgue measure on $R^{1}$. The Fubini theorem implies that for $Q-$ a.e. $u(\cdot ; 0)$ the set of $y_{0}$ satisfying $(7.4)$ has Lebesgue measure zero. This proves (ii). Since two different intervals in $\xi_{t}$ cannot have a common end point, 
there have to be infinitely many such intervals in between. Finally, since $\varphi_{t}$ is a continuous nondecreasing function, and $\varphi_{t}$ is constant on each of these intervals, the range of $\varphi_{t}$ from these intervals has to be dense on its full range.

\section{Behavior of Solutions with Random Initial Data for (1.3)}

In this section we prove an analogous result of Theorem 4 for the nonconservative model (1.3). We use mainly the method of characteristics, an approach potentially more general than the one used in Sect. 7. For the initial data we will assume that $\rho_{0} \in C^{1} \cap L^{1}\left(R^{1}\right)$, and $u(\cdot ; 0)=u_{0}(\cdot)$ is random with distribution $Q$, satisfying $(\mathrm{Q} 1)$, whereas $(\mathrm{Q} 2)$ is replaced by a more stringent condition:

$\left(\mathrm{Q} 2^{\prime}\right)$ There exists a function $\omega(h)$, such that for almost a.e. $u_{0}$,

$$
\begin{aligned}
& \lim _{|h| \rightarrow 0} \sup \frac{u_{0}(y+h)-u_{0}(y)}{\omega(h)}=1, \\
& \lim _{|h| \rightarrow 0} \inf \frac{u_{0}(y+h)-u_{0}(y)}{\omega(h)}=-1,
\end{aligned}
$$

holds for all $y \in R^{1}$, and the convergence is uniform on compact sets. Moreover, $\omega$ satisfies

(1) $\lim _{|h| \rightarrow 0} \omega(h)=0, \quad \lim _{|h| \rightarrow 0} \frac{\omega(h)}{h}=+\infty$.

(2) There exists a constant $\varepsilon>0$, such that for any $h>0$, one can find $h_{1}>0$, such that $\omega\left(h_{1}\right)<\frac{1}{4} \omega(h)$ and $h_{1}>\varepsilon h$.

(2) essentially says that $\omega$ has algebraic behavior near 0 . Obviously, the Wiener process satisfies $\left(\mathrm{Q}^{\prime}\right)$ with $\omega(h)=\left(2 h \log \frac{1}{h}\right)^{1 / 2}$.

Theorem 5. For a.e. $u_{0}$, the weak solution of (1.3) constructed in Sect. 6 has the following properties: for any $t>0$, let $\left\{x_{i}\right\}$ be the set of shock locations at time t. Then

(a) The measure $\rho(\cdot, t)$ is a pure point measure: $\rho(x, t)=\Sigma_{i} m_{i} \delta\left(x-x_{i}\right)$, $m_{i} \geqq 0$.

(b) $\left\{x_{i}\right\}$ is dense in $R^{1}$.

Proof. Take $u(x ; 0)$ such that (Q1) and $\left(\mathrm{Q}^{\prime}\right)$ are satisfied and fix any $t^{*}>0$. We first show that the set of shock locations is dense in $R^{1}$. Assume to the contrary that there exists an interval $\Delta$ on which $u\left(\cdot, t^{*}\right)$ is continuous. Then for any $x \in \Delta$ there is a unique backward characteristic $\xi:\left[0, t^{*}\right] \rightarrow R^{1}$ connecting $x$ to a point $y$ at $t=0$. Furthermore, along $\xi$ we have

$$
\frac{d}{d t} \xi(t)=\bar{u}(t), \quad \frac{d}{d t} h(\xi(t), t)=0, \quad \frac{d}{d t} \bar{u}(t)=-h(\xi(t), t) .
$$

Thus $\xi$ is given by

$$
\xi(t)=y+t u_{0}(y)-h_{0}(y) \frac{t^{2}}{2}, \quad \xi\left(t^{*}\right)=x .
$$

The mapping from $y$ to $x$ is obviously continuous. It is also one-to-one since the characteristics do not intersect between 0 and $t^{*}$. Therefore, the inverse mapping 
from $x$ to $y$ is also well-defined and continuous. Hence, there is a interval $J \in R^{1}$ at $t=0$ on which the forward characteristics, also defined by (8.3), do not intersect between $t=0$ and $t=t^{*}$, i.e. if we take $y_{1}, y_{2} \in J, y_{1}<y_{2}$, then we should also have for $0 \leqq t \leqq t^{*}$,

or

$$
y_{1}+t u_{0}\left(y_{1}\right)-\frac{t^{2}}{2} h_{0}\left(y_{1}\right)<y_{2}+t u_{0}\left(y_{2}\right)-\frac{t^{2}}{2} h_{0}\left(y_{2}\right)
$$

$$
t\left(u_{0}\left(y_{2}\right)-u_{0}\left(y_{1}\right)\right)>y_{1}-y_{2}-\frac{t^{2}}{2}\left(h_{0}\left(y_{1}\right)-h_{0}\left(y_{2}\right)\right) \geqq-C\left(y_{2}-y_{1}\right)
$$

for some constant $C$. This clearly violates $\left(\mathrm{Q}^{\prime}\right)$. Hence we have proved that the set of shock locations is dense in $R^{1}$.

Let $x_{0} \in\left\{x_{i}\right\}$. From $x_{0}$ we draw the minimal and maximal backward characteristics. Assume that they arrive at $y_{1}$ and $y_{2}$ respectively at $t=0$. These characteristics are classical in the sense that (8.2) is satisfied along them. Therefore, we see that $h\left(\cdot, t^{*}\right)$ is discontinuous at $x_{0}$, and

$$
h\left(x_{0}+, t^{*}\right)=h_{0}\left(y_{2}\right), \quad h\left(x_{0}-, t^{*}\right)=h_{0}\left(y_{1}\right) .
$$

In other words, the masses initially distributed in $\left(y_{1}, y_{2}\right)$ are absorbed into $x_{0}$ at $t=t^{*}$.

Let $S\left(u_{0}\right)=\{y$ : There is a genuine (or classical) characteristic connecting $y$ at $t=0$ to some $x$ at $\left.t=t^{*}\right\}$. We want to prove that the Lebesgue measure of $S\left(u_{0}\right)$ is zero.

Let $y_{0} \in S\left(u_{0}\right)$. If $y \in S\left(u_{0}\right), y>y_{0}$, then we must have

$$
y+t u(y ; 0)-\frac{t^{2}}{2} h_{0}(y)>y_{0}+t u\left(y_{0} ; 0\right)-\frac{t^{2}}{2} h_{0}\left(y_{0}\right)
$$

for $0<t<t^{*}$. Equation (8.6) implies that

$$
u(y ; 0)-u\left(y_{0} ; 0\right)>-C_{0}\left(y-y_{0}\right)
$$

for some positive constant $C_{0}$. Choose $y_{1}$ sufficiently close to $y_{0}, y_{1}>y_{0}$ such that

(i) $u\left(y_{1} ; 0\right)-u\left(y_{0} ; 0\right)<-\frac{1}{2} \omega\left(y_{1}-y_{0}\right)$,

(ii) $\left|u(y ; 0)-u\left(y_{1} ; 0\right)\right|<2 \omega\left(y_{1}-y\right)$

for $y \in\left(y_{0}, y_{1}\right)$. Then

$$
u(y ; 0)<2 \omega\left(y_{1}-y\right)+u\left(y_{1} ; 0\right)<2 \omega\left(y_{1}-y\right)-\frac{1}{2} \omega\left(y_{1}-y_{0}\right)+u\left(y_{0} ; 0\right) .
$$

Next we choose $y_{2} \in\left(y_{0}, y_{1}\right)$ such that

$$
\omega\left(y_{1}-y_{2}\right)<\frac{1}{4} \omega\left(y_{1}-y_{0}\right)
$$

and $y_{1}-y_{2} \geqq \varepsilon\left(y_{1}-y_{0}\right)$. For $y \in\left(y_{2}, y_{1}\right)$ we have

$$
\begin{aligned}
& u(y ; 0)-u\left(y_{0} ; 0\right)<2 \omega\left(y_{1}-y_{2}\right)-\frac{1}{2} \omega\left(y_{1}-y_{0}\right) \\
& <-\frac{1}{4} \omega\left(y_{1}-y_{0}\right)<-C_{0}\left(y_{1}-y_{0}\right) \leqq-C_{0}\left(y-y_{0}\right)
\end{aligned}
$$


if $y_{1}$ is sufficiently close to $y_{0}$. Comparing (8.8) with (8.7) we see that $\left(y_{2}, y_{1}\right)$ $\bar{E} S\left(u_{0}\right)$. Therefore, we have

$$
\frac{\left|S\left(u_{0}\right) \cap\left[y_{0}, y_{1}\right]\right|}{\left|\left[y_{0}, y_{1}\right]\right|} \leqq 1-\varepsilon .
$$

Here we used $|A|$ to denote the Lebesgue measure of the set $A$. Hence $y_{0}$ is not a point of density of $S\left(u_{0}\right)$. Since this is true for all $y_{0} \in S\left(u_{0}\right)$, we have $\left|S\left(u_{0}\right)\right|=0$.

Acknowledgements. We thank U. Frisch for numerous stimulating discussions related to the problem discussed in this paper. The work by E was supported by the Sloan Foundation. The work of Yu. Rykov was supported, in part, by the Foundation of Fundamental Research of Russia under the grant N 93-01-16090. The work of Ya. Sinai was partially supported by the National Science Foundation under grant DMS-9404437.

\section{Appendix: Contact Points on the Convex Hull}

In this appendix we summarize some properties of the contact points on the convex hull of $\phi_{1} \circ \phi_{2}^{-1}$, extending the discussions in Sect. 2. We will assume that $P_{0}$ has a density $\rho_{0}(x), u_{0}$ is continuous.

Definition A1. A point $z$ is called special if

$$
\tilde{c}(z, z)=\min _{z^{+} \geqq z} \tilde{c}\left(z, z^{+}\right)=\max _{z^{-} \leqq z} \tilde{c}\left(z^{-}, z\right)=\tilde{c}(z, z) .
$$

Lemma A1. The set of special points is closed.

Proof. Let $z_{n}$ be a sequence of special points, $z_{n} \rightarrow z$. We claim that $z$ is a special point. Assume to the contrary, then for some $z^{-}<z<z^{+}$,

$$
\tilde{c}\left(z^{-}, z\right)>\tilde{c}\left(z, z^{+}\right) .
$$

But $\tilde{c}\left(z^{-}, z\right)=\lim _{n \rightarrow \infty} \tilde{c}\left(z^{-}, z_{n}\right), \tilde{c}\left(z, z^{+}\right)=\lim _{n \rightarrow \infty} \tilde{c}\left(z_{n}, z^{+}\right)$. Therefore, for sufficiently large $n$,

$$
\tilde{c}\left(z^{-}, z_{n}\right)>\tilde{c}\left(z_{n}, z^{+}\right)
$$

which contradicts our assumption that $z_{n}$ is a special point.

Definition A2. A special point $z$ is called single if $\tilde{c}\left(z, z^{+}\right)>\tilde{c}\left(z^{-}, z\right)$ for any $z^{-}<z<z^{+}$. A special point $z$ is called multiple if there exists either $z^{+}>z$ for which $\tilde{c}\left(z^{+}, z\right)=\tilde{c}(z, z)$, or $z^{-}<z$ for which $\tilde{c}\left(z^{-}, z\right)=\tilde{c}(z, z)$.

For every $z$ consider a segment $\left[z_{0}^{-}, z_{0}^{+}\right]$, where $z_{0}^{-}=\min \{y \leqq z: \tilde{c}(y, z)=$ $\tilde{c}(z, z)\}, z_{0}^{+}=\max \{y \geqq z: \tilde{c}(z, y)=\tilde{c}(z, z)\}$. If a point $z$ is multiple then $\left[z_{0}^{-}, z_{0}^{+}\right]$ has positive length.

Lemma A2. Take $y \in\left(z, z_{0}^{+}\right)$where $\tilde{c}(z, y)>\tilde{c}(z, z)$. Then $y$ is not a special point.

Proof. We have

$$
\tilde{c}\left(z, z_{0}^{+}\right)=p_{1} \tilde{c}(z, y)+p_{2} \tilde{c}\left(y, z_{0}^{+}\right)
$$


where

$$
p_{1}=\frac{\int_{z}^{y} \rho_{0}(\eta) d \eta}{\int_{z}^{z_{0}^{+}} \rho_{0}(\eta) d \eta}, \quad p_{2}=\frac{\int_{y}^{z_{0}^{+}} \rho_{0}(\eta) d \eta}{\int_{z}^{z_{0}^{+}} \rho_{0}(\eta) d \eta} .
$$

Since $\tilde{c}(z, y)>\tilde{c}\left(z, z_{0}^{+}\right)$, we conclude that $\tilde{c}\left(y, z_{0}^{+}\right)<\tilde{c}(z, y)$. This contradicts the definition of special point.

In the same way one proves the following lemma.

Lemma $\mathbf{A 2} 2^{\prime}$. If $y \in\left(z_{0}^{-}, z\right)$ and $\tilde{c}(y, z)<\tilde{c}(z, z)$ then $y$ is not a special point.

Lemma A3. Take any point $z^{\prime} \in\left[z, z_{0}^{+}\right]$where $\tilde{c}\left(z, z^{\prime}\right)=\tilde{c}(z, z)$, then $z^{\prime}$ is a special point.

Proof. We shall consider two cases.

1) $z<z^{-}<z^{\prime}, z^{\prime}<z^{+}$. As above, we have

$$
\tilde{c}(z, z)=\tilde{c}\left(z, z^{\prime}\right)=p_{1} \tilde{c}\left(z, z^{-}\right)+p_{2} \tilde{c}\left(z^{-}, z^{\prime}\right)=\min _{y \geqq z} \tilde{c}(z, y)
$$

for some $p_{1}, p_{2} \geqq 0, \quad p_{1}+p_{2}=1$, and $\tilde{c}\left(z, z^{\prime}\right) \leqq \tilde{c}\left(z, z^{-}\right)$. We conclude that $\tilde{c}\left(z^{-}, z^{\prime}\right) \leqq \tilde{c}\left(z, z^{\prime}\right)$. Also for some $p_{1}^{\prime}, p_{2}^{\prime} \geqq 0, p_{1}^{\prime}+p_{2}^{\prime}=1$,

$$
\tilde{c}\left(z, z^{+}\right)=p_{1}^{\prime} \tilde{c}\left(z, z^{\prime}\right)+p_{2}^{\prime} \tilde{c}\left(z^{\prime}, z^{+}\right) \geqq \tilde{c}\left(z, z^{\prime}\right) .
$$

We conclude that $\tilde{c}\left(z^{\prime}, z^{+}\right) \geqq \tilde{c}\left(z, z^{\prime}\right)$, i.e.

$$
\tilde{c}\left(z^{-}, z^{\prime}\right) \leqq \tilde{c}\left(z, z^{\prime}\right) \leqq \tilde{c}\left(z^{\prime}, z^{+}\right) .
$$

2) $z^{-}<z, z^{\prime}<z^{+}$.

We have for some $p_{1}^{\prime \prime}, p_{2}^{\prime \prime} \geqq 0, p_{1}^{\prime \prime}+p_{2}^{\prime \prime}=1$,

$$
\tilde{c}\left(z^{-}, z^{\prime}\right)=p_{1}^{\prime \prime} \tilde{c}\left(z^{-}, z\right)+p_{2}^{\prime \prime} \tilde{c}\left(z, z^{\prime}\right) .
$$

Since $\tilde{c}\left(z, z^{\prime}\right)=\tilde{c}(z, z), \tilde{c}\left(z^{-}, z\right) \leqq \tilde{c}\left(z, z^{\prime}\right)$, we find that $\tilde{c}\left(z^{-}, z^{\prime}\right) \leqq \tilde{c}\left(z, z^{\prime}\right)$, while we have already shown that $\tilde{c}\left(z^{\prime}, z^{+}\right) \geqq \tilde{c}\left(z, z^{\prime}\right)$.

The lemma is proven.

Lemma A3'. Take any point $z^{\prime} \in\left[z_{0}^{-}, z\right)$, where $\tilde{c}\left(z^{\prime}, z\right)=\tilde{c}(z, z)$. Then $z^{\prime}$ is a special point.

Take the segment $\left[z_{0}^{-}, z_{0}^{+}\right]$constructed for the point $z$. We shall denote the dependence on $z$ as $\left[z_{0}^{-}, z_{0}^{+}\right]_{z}$.

Lemma A.4. If $z^{\prime}$ is a special point $z^{\prime} \in\left[z_{0}^{-}, z_{0}^{+}\right]_{z}$, then $\left[z_{0}^{-}, z_{0}^{+}\right]_{z^{\prime}}=\left[z_{0}^{-}, z_{0}^{+}\right]_{z}$.

Proof. We claim that $\tilde{c}(z, z)=\tilde{c}\left(z^{\prime}, z^{\prime}\right)$. Indeed, $\tilde{c}\left(z, z^{\prime}\right)=\tilde{c}(z, z)$ according to Lemmas $2,2^{\prime}, 3,3^{\prime}$. So the function $\tilde{c}(z, y)$ as a function of $y$ attains its extremum on the semi-line at $z^{\prime} . z^{\prime}$ is an interior point of the semi-line. So $\left.\frac{\partial}{\partial y} \tilde{c}(z, y)\right|_{y=z^{\prime}}=0$. This gives

$$
\left[z^{\prime}+t u_{0}\left(z^{\prime}\right)\right] \int_{z}^{z^{\prime}} \rho_{0}(\eta) d \eta=\int_{z}^{z^{\prime}}\left(\eta+t u_{0}(\eta)\right) \rho_{0}(\eta) d \eta,
$$

and so $\tilde{c}\left(z^{\prime}, z^{\prime}\right)=\tilde{c}\left(z^{\prime}, z\right)=\tilde{c}(z, z)$. 
Suppose $z^{\prime}<z$. We have, for some $p_{1}, p_{2} \geqq 0, p_{1}+p_{2}=1$,

$$
\begin{gathered}
\tilde{c}(z, z)=\tilde{c}\left(z_{0}^{-}, z\right)=p_{1} \tilde{c}\left(z_{0}^{-}, z^{\prime}\right)+p_{2} \tilde{c}\left(z^{\prime}, z\right)=p_{1} \tilde{c}\left(z_{0}^{-}, z^{\prime}\right)+p_{2} \tilde{c}(z, z), \\
\tilde{c}\left(z_{0}^{-}, z^{\prime}\right)=\tilde{c}(z, z)=\tilde{c}\left(z^{\prime}, z^{\prime}\right) .
\end{gathered}
$$

If we have $z^{-}<z_{0}^{-}$such that $\tilde{c}\left(z^{-}, z^{\prime}\right)=\tilde{c}\left(z^{\prime}, z^{\prime}\right)$, then for some $p_{1}^{\prime}, p_{2}^{\prime} \geqq 0, p_{1}^{\prime}+$ $p_{2}^{\prime}=1$,

$$
\tilde{c}\left(z^{-}, z\right)=p_{1}^{\prime} \tilde{c}\left(z^{-}, z^{\prime}\right)+p_{2}^{\prime} \tilde{c}\left(z^{\prime}, z\right)=p_{1}^{\prime} \tilde{c}\left(z^{\prime}, z\right)+p_{2}^{\prime} \tilde{c}(z, z)=\tilde{c}(z, z) .
$$

That contradicts our assumption that $z_{0}^{-}$is the smallest with such a property. The analogous calculations can be done with $z_{0}^{+}$.

The case $z<z^{\prime}$ can be analyzed in a similar way.

\section{References}

[BG] Brenier, Y., Grenier, E.: On the model of pressureless gases with sticky particles. Preprint, 1995

[CPY] Carnevale, G.F., Pomeau, Y., Young, W.R.: Statistics of ballistic agglomeration. Phys. Rev. Lett., 64, no. 24, 2913 (1990)

[D] Dafermos, C.: Generalized characteristics and the structure of solutions of hyperbolic conservation laws. Indiana Univ. Math. J. 26, 1097-1119 (1977)

[GMS] Gurbatov, S.N., Malakhov, A.N., Saichev, A.I.: Nonlinear Random Waves and Turbulence in Nondispersive Media: Waves, Rays and Particles. Manchester: Manchester University Press, 1991

[KPS] Kofman, L., Pogosyan, D., Shandarin, S.: Structure of the universe in the two-dimensional model of adhesion. Mon. Nat. R. Astr. Soc. 242, 200-208 (1990)

[L] Lax, P.D.: Hyperbolic systems of conservation laws: II. Comm. Pure. Appl. Math. 10, 537-556 (1957)

[O] Oleinik, O.A.: Discontinuous solutions of nonlinear differential equations. Uspekhi Mat. Nauk. 12, 3-73 (1957)

[P] Peebles, P.J.E.: The Large Scale Structures of the Universe. Princeton, NJ: Princeton University Press, 1980

[SZ] Shandarin, S.F., Zeldovich, Ya.B.: The large-scale structures of the universe: Turbulence, intermittency, structures in a self-gravitating medium. Rev. Mod. Phys. 61, 185-. 220 (1989)

[SAF] She, Z.S., Aurell, E., Frisch, U.: The inviscid Burgers equation with initial data of Brownian type. Commun. Math. Phys. 148, 623-641 (1992)

[S] Sinai, Ya.G.: Statistics of shocks in solutions of inviscid Burgers equation. Commun. Math. Phys. 148, 601-622 (1992)

[VDFN] Vergassola, M., Dubrulle, B., Frisch, U., Noullez, A.: Burgers' equation, devil's staircases and the mass distribution function for large-scale structures. Astron \& Astrophys 289, 325-356 (1994)

[V] Volpert, A.I.: The space BV and quasilinear equations. Math. USSR-Sbornik. 2, 225-267 (1967)

[Z] Zeldovich, Ya.B.: Gravitational instability: An approximate theory for large density perturbations. Astron \& Astrophys. 5, 84-89 (1970) 